\title{
Laminin-Dependent Interaction between Astrocytes and Microglia
}

\section{A Role in Retinal Angiogenesis}

\author{
Saptarshi Biswas, ${ }^{* \dagger \ddagger}$ Galina Bachay, ${ }^{* \dagger \ddagger}$ Julianne Chu, ${ }^{\dagger}$ Dale D. Hunter, ${ }^{* \dagger}$ and William J. Brunken ${ }^{* \dagger}$
}

From the Department of Ophthalmology, ${ }^{*}$ State University of New York Upstate Medical University, Syracuse; the Department of Ophthalmology, ${ }^{\dagger}$ State University of New York Downstate Medical Center, Brooklyn; and the Center for Vision Research, ${ }^{\ddagger}$ Syracuse, New York

\author{
Accepted for publication \\ May 31, 2017. \\ Address correspondence to \\ William J. Brunken, Ph.D., \\ SUNY Upstate Medical \\ University, Department of \\ Ophthalmology, NRB 3601, \\ 505 Irving Ave, Syracuse, NY \\ 13210. E-mail: brunkenw@ \\ upstate.edu.
}

\begin{abstract}
Retinal vascular diseases are among the leading causes of acquired blindness. In recent years, retinal microglia have been shown to influence vascular branching density and endothelial cell proliferation. However, how microglial recruitment and activation are regulated during development remains unclear. We hypothesized that microglial recruitment, activation, and down-stream signaling are modulated by components of the mural basement membrane. We used a reverse genetic approach to disrupt laminin expression in the vascular basement membrane and demonstrate that microglia respond to the mural basement membrane in an isoform-specific manner. Microglial density is significantly increased in the laminin $\gamma$ 3-null $\left(\mathrm{LamC}^{-/-}\right)$retinal superficial vascular plexus and consequently the vascular branching density is increased. Microglia also respond to astrocyte-derived matrices and become hyperactivated in the $\mathrm{LamC}^{-/-}$retina or when tested in vitro with cell-derived matrix. Pharmacological activation of microglia in the wild-type retina produced an $\mathrm{LamC3}^{-/-}$-like vascular phenotype, whereas pharmacological blocking of microglial activation in the $2 a m c 3^{-/-}$retina rescued the wild-type vascular phenotype. On the molecular level, microglial transforming growth factor- $\beta 1$ expression is downregulated in the $\mathrm{LamC}^{-/-}$retina, and SMAD signaling decreased in endothelial cells with a consequent increase in endothelial proliferation. The reverse effects were seen in the Lamb2 $2^{-/}$retina. Together, our results demonstrate a novel mechanism by which laminins modulate vascular branching and endothelial cell proliferation during retinal angiogenesis. (Am J Pathol 2017, 187: 2112-2127; http://dx.doi.org/10.1016/j.ajpath.2017.05.016)
\end{abstract}

Disruptions in the mechanisms that govern retinal angiogenesis are the leading cause of adult blindness in the developed world. ${ }^{1}$ As the mouse retina vascularizes entirely postnatally, it has become an established model to elucidate mechanisms of retinal angiogenesis. In the mouse retina, a superficial vascular plexus (SVP) emerges from the central artery and grows out over the retinal surface, following an astrocyte template between postnatal days 1 and 8 (P1 to P8). Vascular sprouts from the SVP dive into the retina, forming a deep capillary plexus at the outer plexiform layer between P7 and P12. Subsequently, an intermediate plexus forms in the inner plexiform layer, completing vascular development by $\mathrm{P} 21$. $^{2}$
Retinal angiogenesis depends on a variety of factors, including soluble growth factors, cell-cell interactions, and cell-extracellular matrix (ECM) interactions. Microvascular basement membrane (BM) in the central nervous system (CNS) consists of two separate layers: an endothelial

\footnotetext{
Supported by NIH/National Eye Institute grant EY126776 (W.J.B.) and a Research to Prevent Blindness unrestricted grant (Departments of Ophthalmology at State University of New York Upstate Medical University and State University of New York Downstate Medical Center).

Disclosures: None declared.

Portions of the data herein were presented at the Association for Research in Vision and Ophthalmology Annual Meeting, May 6, 2014, Orlando, FL, and May 3, 2015, Denver, CO.
} 
cell-derived BM and an astrocyte-derived BM; these fused BMs ensheath the vascular tube. ${ }^{3}$ Laminins, a major component of the BM, have been shown to modulate retinal angiogenesis. ${ }^{4,5}$ Laminins are heterotrimeric glycoproteins made of $\alpha-, \beta$-, and $\gamma$-chains. Five $\alpha$-, three $\beta$-, and three $\gamma$-chains have so far been identified, combining into 16 different heterotrimers, ${ }^{6,7}$ and have overlapping, yet distinct, tissue distributions and functions. ${ }^{8,9}$ In the vasculature, endothelial cell-derived laminins ( $\alpha 4-$ and $\alpha 5$-containing isoforms) and astrocyte-derived laminins ( $\alpha 2$-containing isoforms) are distinct isoforms and have distinct contributions to CNS vascular function. ${ }^{3}$

Microglia are the resident macrophages of the CNS. Microglia populate retina at an early embryonic age, well before astrocytes or vasculature. ${ }^{10,11}$ Previous studies reported that microglia regulate vascular branching by facilitating vascular anastomosis ${ }^{10,12}$ as well as regulating the turning of vascular sprouts in the deep plexus. ${ }^{13}$ In addition, microglia secrete proangiogenic factors in their activated state, whereas resting microglia secrete antiangiogenic factors. ${ }^{14}$ However, little is known about how microglial recruitment and activation are regulated during developmental angiogenesis in the retina.

Microglia interact with the astrocyte-derived ECM during migration, ${ }^{15}$ but the physiological consequence of this interaction is yet undetermined. Using reverse genetic approaches, we have begun to explore the mechanisms of microglial recruitment and activation in the retina. Previously, we reported that astrocyte migration and template formation are dependent on inner limiting membrane laminins. ${ }^{5}$ Moreover, deletion of the Lamc3 gene (laminin $\gamma 3$-chain) resulted in a hyperbranched vascular plexus, and preliminary data suggested that microglia were recruited in greater numbers to the vascular front in $\mathrm{Lamc}^{-1-}$ retinas. $^{5}$ From these preliminary results, we hypothesized that $\gamma 3$-containing laminins in the microvascular BM influence microglial recruitment and activation in the retina, and thereby play an important role in vascular patterning.

In this study, we critically test this hypothesis and show that in the $L a m c 3^{-1-}$ retina, microglial density is increased specifically around the nascent SVP, where they are associated with mature astrocytes, producing an increase in the vascular branching density. We further demonstrate that the interaction with astrocyte-derived ECM via $\beta 1$-containing integrin influences microglial activation. In the absence of $\gamma 3$-containing laminins, microglia are hyperactivated; microglial transforming growth factor (TGF)- $\beta 1$ expression is down-regulated, and SMAD3-mediated signaling in the endothelial cells is decreased. Consequently, endothelial cell proliferation is increased in the nascent plexus of the Lamc $3^{-1-}$ retina. Pharmacological activation of microglia in the wild-type (WT) retina produced an $\mathrm{Lamc}^{-{ }^{-}-}$-like vascular phenotype, whereas pharmacological blocking of microglial activation in the Lamc $^{-1-}$ retina produced a WT-like vascular phenotype. Finally, our results suggest that different laminin isoforms vary in their effects on microglial TGF- $\beta 1$ expression and endothelial cell proliferation.

Together, our results suggest that the $\gamma 3$-containing laminins in the vascular BM restrict vascular branching and endothelial cell proliferation during retinal angiogenesis. Our results suggest a novel regulation of retinal angiogenesis by a laminin-dependent mechanism of microglial recruitment and activation.

\section{Materials and Methods}

\section{Mice}

All procedures involving animals were performed in accordance with the Animal Care and Use Committees of State University of New York Downstate Medical Center (Brooklyn, New York) and State University of New York Upstate Medical University (Syracuse, New York). Targeted deletions of the laminin $\beta 2$-gene (Lamb2) and laminin $\gamma 3$-gene (Lamc3) have been described previously. ${ }^{16-19}$ The laminin $\gamma 3$-null and laminin $\beta 2$-null mice strains were backcrossed over nine generations to $\mathrm{C} 57 \mathrm{bl} / 6 \mathrm{~J}$. There was no rd1 and $\mathrm{rd} 8$ mutation in our mice strains. The notations used for the genotypes are as follows: laminin $\gamma 3$-null or $L a m c 3^{-/-}$and laminin $\beta 2$-null or Lamb2 $2^{-1-}$. For our experiments, times of birth and tissue harvest (early morning in both cases) were kept consistent between nonlittermate WT and Lamc $^{-1-}$ pups.

\section{Immunohistochemistry}

To prepare retinal whole mounts, eyes were enucleated and fixed in $4 \%$ paraformaldehyde for 10 minutes; retinas were dissected, flat mounted, and treated with absolute methanol at $-20^{\circ} \mathrm{C}$ for 10 minutes. In case of the whole mount preparation for TGF- $\beta 1$ staining, the eyes were fixed in $2 \%$ paraformaldehyde for 5 minutes. After a phosphate-buffered saline wash, retinas were incubated overnight at $4{ }^{\circ} \mathrm{C}$ in the blocking buffer (5\% goat or donkey serum and $0.3 \%$ Triton $\mathrm{X}-100)$. The retinas were then incubated with primary antibodies in antibody diluting solution (5\% goat/donkey serum and $0.01 \%$ Triton $\mathrm{X}-100$ ) for 48 hours at $4^{\circ} \mathrm{C}$, washed, and incubated with secondary antibodies for 24 hours. Samples were imaged using a Orca-R2 camera (Hamamatsu Ltd., Hamamatsu City, Japan) with a E800 microscope (Nikon Ltd., Tokyo, Japan). The Volocity software package version 6.3 (Perkin-Elmer, Waltham, MA) was used to acquire, process, and analyze images.

\section{Reagents}

Primary antibodies used were rabbit anti-laminin (Sigma, St. Louis, MO; 1:500), rabbit anti-laminin $\gamma 3$ (R96: our laboratory $\left.^{5,19} ; 1: 10,000\right)$, rabbit anti-glial fibrillary acidic protein (Chemicon, Billerica, MA; 1:500), rat anti-CD31 (Chemicon; 1:250), fluorescein isothiocyanate-conjugated isolectin B4 (Sigma; 1:250), rat anti-F4/80 (Life 
Technologies, Grand Island, NY; 1:250), rabbit anti-Iba1 (Wako Laboratory Chemicals, Cape Charles, VA; 1:300), rat anti-CD68 (AbD Serotec, Raleigh, NC; 1:100), rat antiphospho-histoneH3 ${ }^{\text {pSer28 }}$ (Sigma; 1:1000), mouse anti-TGF- $\beta 1$ (R\&D Systems, Minneapolis, MN; 1:50), rabbit anti-phospho-SMAD3 (Abcam, Cambridge, MA; 1:250), rat anti-CD45 (Santa Cruz Biotechnology, Dallas, TX; 1:250), rat anti-platelet-derived growth factor receptor$\alpha$ (BD Biosciences, San Jose, CA; 1:500), and rabbit anti-Pax2 (Abcam; 1:500). For secondary antibodies, goat anti-rabbit 488 and 594, goat anti-rat 488 and 568, donkey anti-rabbit 488, 594, and 647, and donkey anti-rat 594 (Life Technologies) were used. Additional reagents used were integrin $\beta 1$-blocking antibody (BD Biosciences, San Jose, CA), papain dissociation system (Worthington Biochemical Corp., Lakewood, NJ), lipopolysaccharide (LPS; Sigma), minocycline hydrochloride (Sigma), SB-431542 (Sigma), and poly-L-lysine (Sigma).

\section{Retinal Astrocyte Isolation}

Astrocytes were isolated from P2 WT and $\mathrm{Lamc}^{-/-}$retinas, as described previously, ${ }^{5}$ with modifications. In brief, six retinas for each genotype were digested with the papain dissociation system (Worthington Biochemical Corp.) for 30 minutes at $37^{\circ} \mathrm{C}$ in $5 \% \mathrm{CO}_{2}$. Digested retinas were titurated with a pipette; dissociated cells were spun and resuspended in Dulbecco's modified Eagle's medium containing
$10 \%$ bovine calf serum and $1 \%$ penicillin/streptomycin. The resuspended cells were plated in $25-\mathrm{cm}^{2}$ tissue culture flasks (Becton, Dickinson and Company, Franklin lakes, NJ) and allowed to grow for 3 days. The flasks were shaken for 4 hours to detach other cell types, and fresh medium was added to the flasks. From here onward, the medium in the flasks was changed every 72 hours, and the cells were allowed to grow for 12 days. The obtained cells were $>95 \%$ astrocytes, judged by the platelet-derived growth factor receptor- $\alpha$ staining.

\section{Primary Microglia Culture}

Primary microglia isolated from P2 WT rat brain were obtained from ScienCell Research Laboratories (Carlsbad, CA). To culture primary microglia, a $25-\mathrm{cm}^{2}$ tissue culture flask was coated with $10 \mathrm{mg} / \mathrm{mL}$ poly-L-lysine. The microglial cells were resuspended in microglia medium (ScienCell Research Laboratories), containing 5\% fetal bovine serum, $1 \%$ microglia growth supplement (ScienCell Research Laboratories), and $1 \%$ penicillin/streptomycin solution in basal medium. The resuspended cells were plated onto a poly-L-lysine-coated flask and grown for 48 hours. The medium in the flask was changed every 72 hours.

To grow microglia in the astrocyte-derived ECM, WT astrocytes were cultured on the coverslips for 4 days, followed by $100 \%$ dimethyl sulfoxide treatment for 1 hour to decellularize the coverslips. The coverslips were washed
A
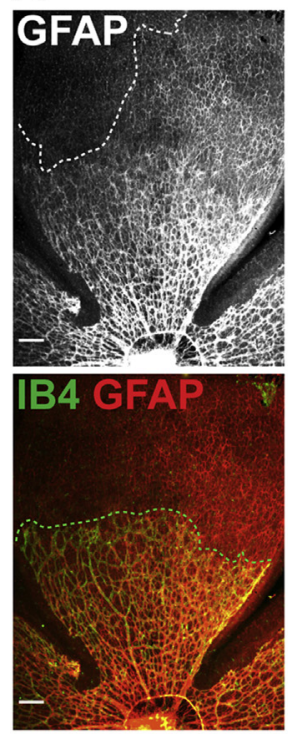

P3 WT
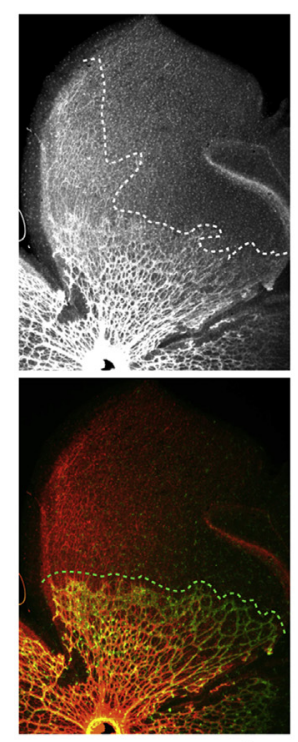

P3 Lamc3 ${ }^{-/-}$
B
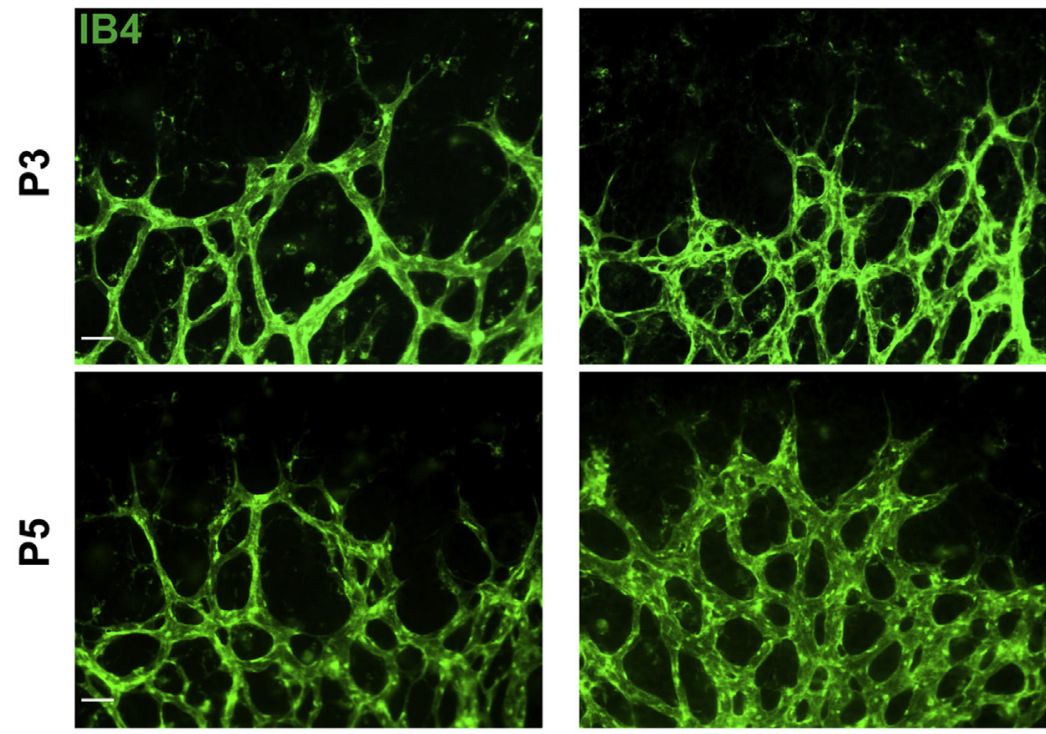

Lamc $^{-/-}$

Figure $1 \mathrm{LamC}^{-/-}$retinas have a hyperbranched SVP. A: P3 WT and $\mathrm{LamC}^{-1-}$ retinal whole mounts were stained with anti-glial fibrillary acidic protein (GFAP; white, top row; red, bottom row) antibody and isolectin B4 (IB4; green). White dashed lines indicate the extent of mature astrocyte template migration. Green dashed lines indicate the extent of vascular tree migration. Note the delayed mature astrocyte migration and vascular tree expansion in the Lamc $^{-/-}$retina (quantification previously reported ${ }^{5}$ ). Times of birth and tissue harvest (early morning in both cases) were kept consistent between nonlittermate WT and $\mathrm{Lamc}^{-/-}$pups. Retinas from at least three mice from each genotype were analyzed. B: P3 and P5 WT and Lamc3 ${ }^{-1-}$ retinal whole mounts were stained with IB4 (green). Scale bars: $160 \mu \mathrm{m}(\mathbf{A}) ; 32 \mu \mathrm{m}$ (B). 
A

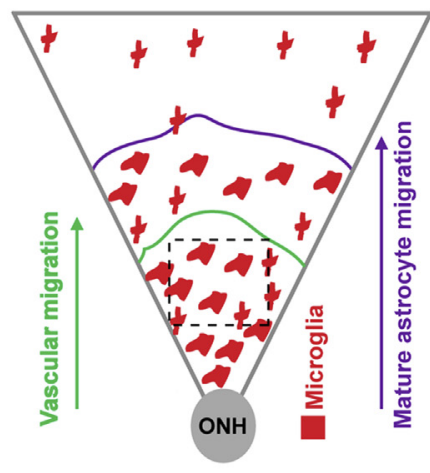

P3

D

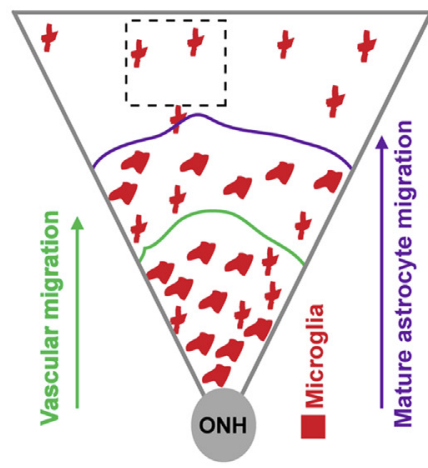

P3
B

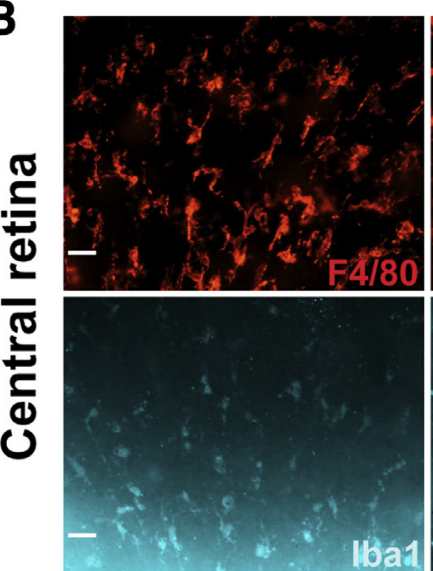

P3 WT

E

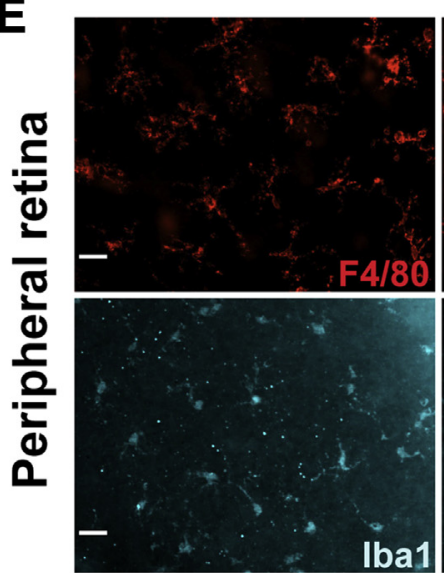

P3 WT

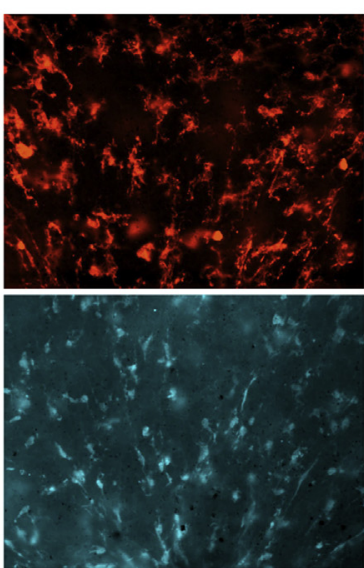

P3 Lamc3 $^{-/-}$

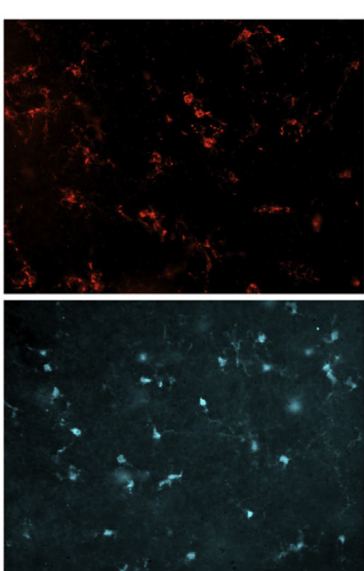

P3 Lamc 3/-

\section{Central}

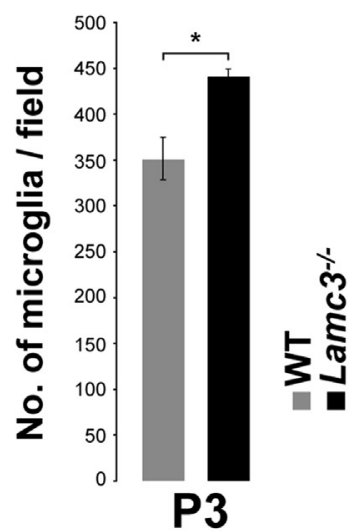

F

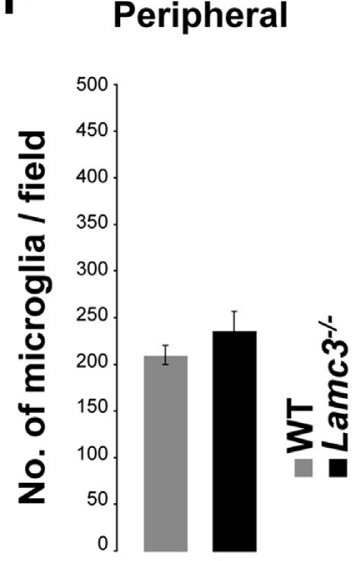

P3

Figure 2 Microglial density increases around the $\mathrm{LamC}^{-1-}$ retinal SVP. A: Schematic of the central retinal region (in the presence of mature astrocyte template and SVP). Images in B were acquired from the boxed area. B: P3 WT and Lamc3 ${ }^{-/-}$retinal whole mounts were stained with anti-F4/80 (red) and antiIba1 (cyan) antibodies. Central retinal images are displayed. C: Quantification of microglial density in P3 WT and Lamc $3^{-/-}$central retinas. D: Schematic of the peripheral retinal region (in the absence of mature astrocyte template and SVP). Images in $\mathbf{E}$ were acquired from the boxed area. E: P3 WT and Lamc3 ${ }^{-1-}$ retinal whole mounts were stained with anti-F4/80 (red) and anti-Iba1 (cyan) antibodies. Peripheral retinal images are displayed. F: Quantification of microglial density in P3 WT and $\mathrm{Lamc}^{-/-}$peripheral retinas. Data are expressed as means \pm SEM. $n=3(\mathbf{C}$ and $\mathbf{F})$. ${ }^{\star} P<0.05$. Scale bars $=32 \mu \mathrm{m}(\mathbf{B}$ and $\mathbf{E})$. $\mathrm{ONH}$, optic nerve head.

three times in microglia medium; then, WT microglia were seeded onto the WT astrocyte-derived ECM. To block microglial integrin $\beta 1$ signaling, either heat-killed or undenatured integrin $\beta 1$-blocking antibody $(25 \mu \mathrm{g} / \mathrm{mL})$ was added to each coverslip. Microglia were allowed to grow for 2 more days.

\section{Astrocyte-Microglia Co-Culture}

For astrocyte-microglia co-culture assay, the WT and Lamc $3^{-/-}$astrocytes were detached with trypsin and replated separately onto glass coverslips in 24-well culture plates (Corning Life Sciences, Tewksbury, MA). Microglia medium $(300 \mu \mathrm{L})$ was added to each coverslip. The astrocytes were grown on the coverslips for 2 days; then, WT microglia were seeded on the astrocytes at a concentration of $10,000 \mathrm{cells} / \mathrm{cm}^{2}$. Fresh microglia medium was added to these coverslips. Microglia and astrocytes were allowed to grow for 2 more days.

\section{In Vivo Injections}

For LPS treatment, P2 WT mice were given a single i.p. injection of LPS dissolved in physiological saline $(1 \mathrm{mg} / \mathrm{kg}$ body weight). The injected mice were euthanized at P5, and their retinas were dissected. For minocycline treatment, $\operatorname{Lamc}^{-/-}$ or WT mice were given three i.p. injections (one injection/day on $\mathrm{P} 0, \mathrm{P} 2$, and $\mathrm{P} 4$ ) of minocycline hydrochloride dissolved in physiological saline (50 mg/kg body weight). The injected mice were euthanized at P5, and their retinas were dissected. For SB-431542 treatment, P3 WT mice were given a single i.p. injection of SB-431542 dissolved in physiological saline $(10 \mathrm{mg} / \mathrm{kg}$ body weight). The injected mice were euthanized at $\mathrm{P} 5$, and their retinas were dissected. 


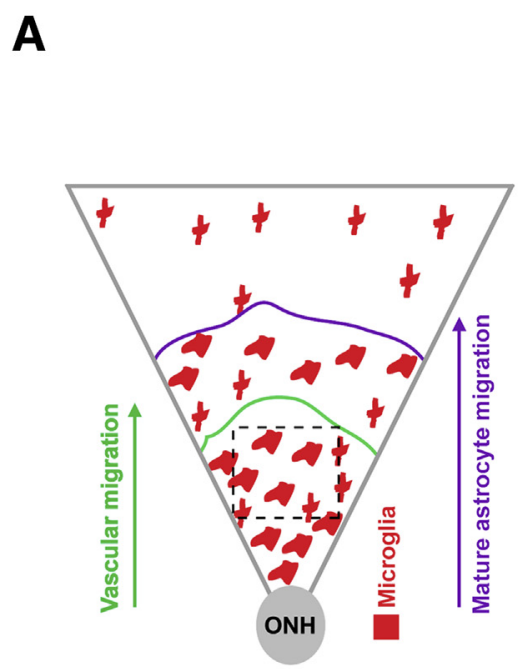

P3
B

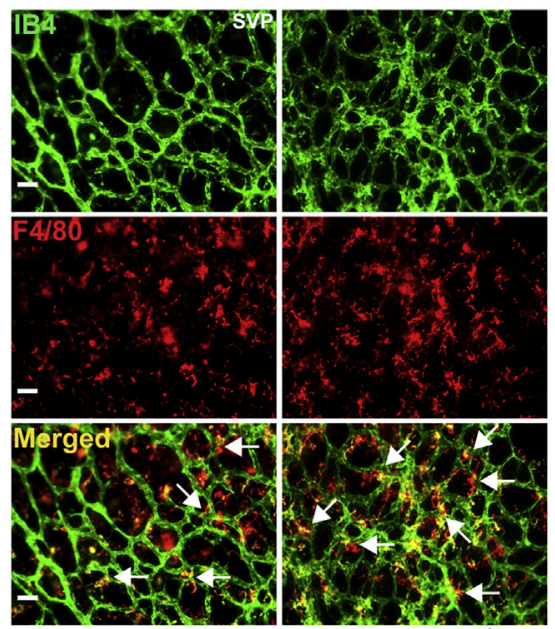

P3 WT
C

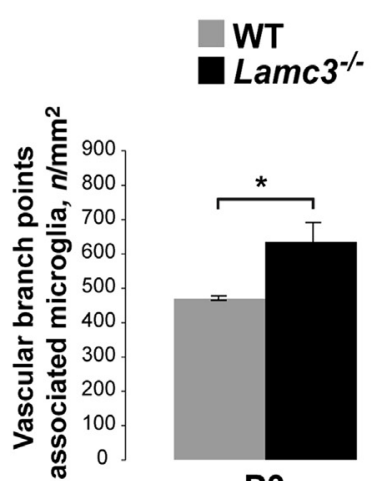

P3
D Saline

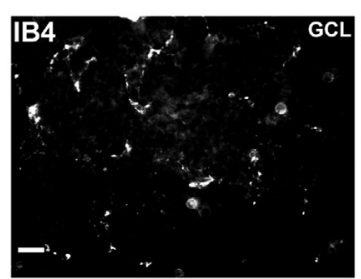

LPS

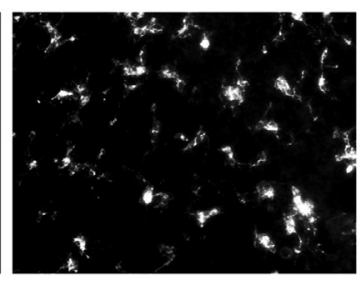

P5 WT

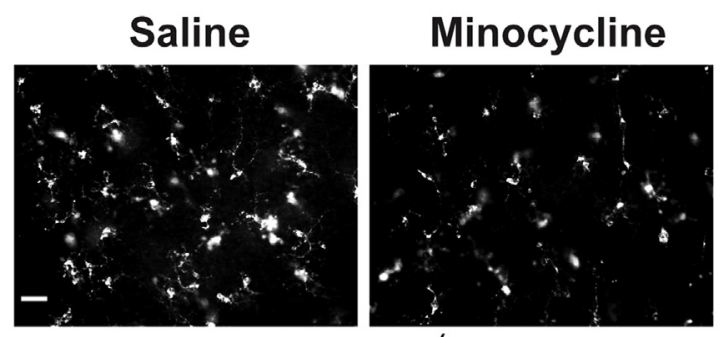

P5 Lamc3-/-

F Saline

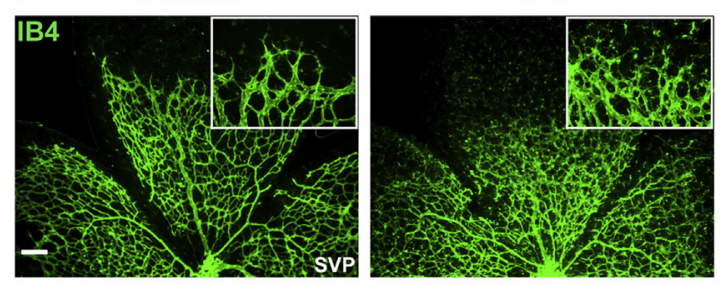

P5 WT

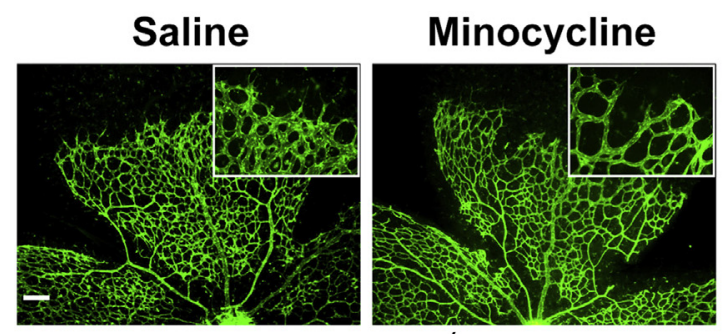

P5 Lamc3 ${ }^{-/-}$
E

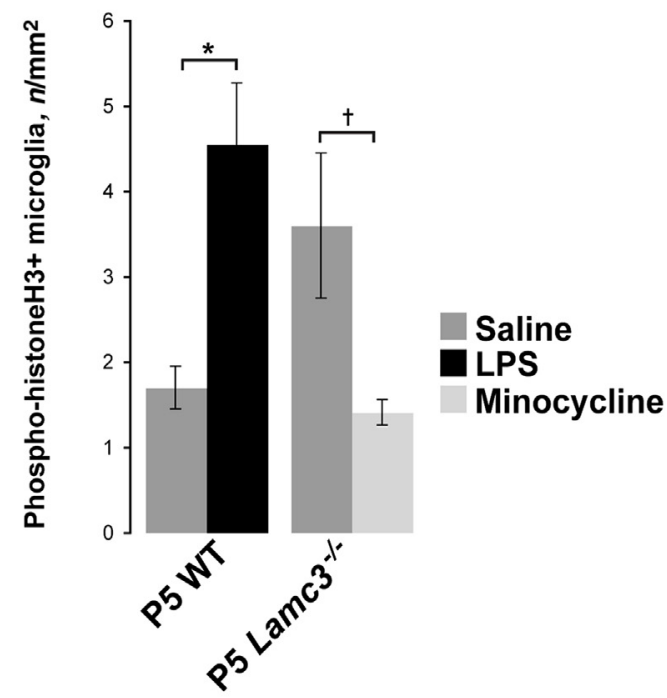

G

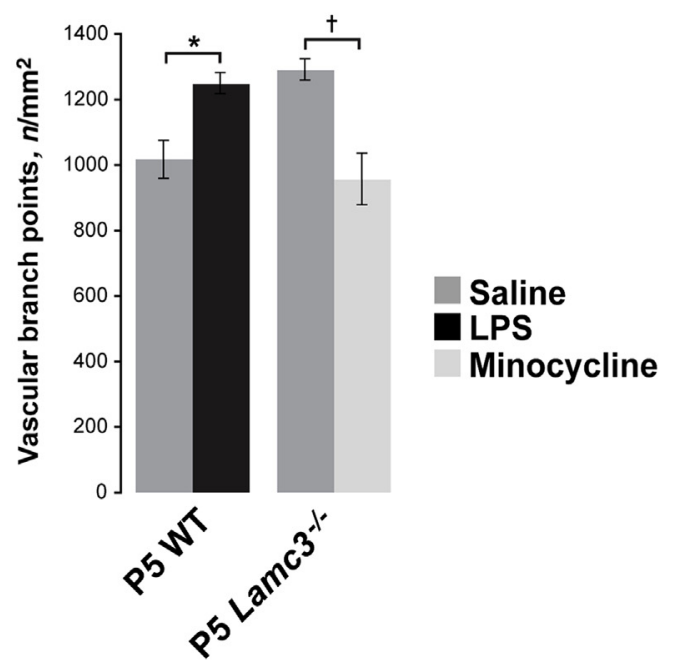




\section{Measurements and Statistical Analysis}

All measurements, unless otherwise specified, were performed using Volocity software version 6.3. For these experiments, data were not segregated based on sex of the animals. In each analysis, measurements were made in all four quadrants of the retina and averaged. For microglial TGF- $\beta 1$ expression in vivo, individual microglial cells were selected from an image and Pearson correlation analysis was performed. For percentage of activated $\left(\mathrm{CD}^{+} 8^{+}\right)$microglia quantification in vitro, three representative fields from each replicate were measured. At least three samples/replicates were used for each statistical analysis. To test for statistical significance of any differences, a $t$-test was performed and a $P<0.05$ was considered statistically significant.

\section{Results}

\section{Lamc $^{-/-}$Retinal SVP Hyperbranching Is Accompanied by a Specific Increase in Microglial Density}

We previously reported that the rate of astrocyte migration dictates the extent of vascular tree expansion. ${ }^{5}$ In P3 WT retinas, the glial fibrillary acidic protein ${ }^{+}$mature astrocyte template has migrated more than halfway across the retinal surface, followed closely by the expanding vascular tree (Figure 1A). In $\mathrm{Lamc}^{-/-}$retinas, the wave of astrocyte migration is slowed with a concomitant delay in vascular expansion (Figure 1A); we previously published that astrocyte migration is delayed by approximately $25 \%$, with a $40 \%$ delay in vascular expansion. ${ }^{5}$ However, we found that the slowly expanding SVP in $L a m c 3^{-1-}$ retinas has a higher branching density than WT controls (Figure 1B) at both P3 and P5 (Figure 1B).

Next, we asked if the astrocyte density was altered in Lamc $3^{-1-}$ retinas, as an increased astrocyte density might account for increased vascular branching. We examined astrocyte density around the growing SVP (Supplemental Figure S1A) using two markers: glial fibrillary acidic protein (labels mature astrocytes) and Pax2 (labels astrocyte nuclei) (Supplemental Figure S1B). However, we failed to observe any significant difference in astrocyte density around the Lamc3 ${ }^{-1-}$ SVP (Supplemental Figure S1, B and C). Together, these results suggest that astrocytes are unlikely to be the direct cause of the hyperbranched phenotype in the $\mathrm{Lamc}^{-1-}$ SVP.
As retinal microglia have been reported to regulate vascular branching density by facilitating anastomosis, ${ }^{10}$ we asked whether microglial density was affected in the $\mathrm{Lamc}^{-1-}$ retina. For our analyses, we measured microglial density in two separate retinal regions at P3: around the vascular tree in the central retina (Figure 2A) and in the absence of vasculature in the peripheral retina (Figure 2D). Two markers, F4/80 and Iba1, were used to label microglia (Figure 2, B and E). Using either of these markers, we observed a significantly higher microglial density in Lamc $^{-/-}$central retinas compared to the WT controls (Figure 2, B and C).

To confirm that this increased density is from the resident pool of intrinsic microglia and not the result of an increase in the invasion of circulating macrophages, CD45 was used as an unequivocal marker of the latter cell type. Vascular macrophages show high expression of CD45, whereas microglia show low CD45 reactivity. ${ }^{20}$ There was no observable difference in CD45 $5^{\text {high }}$ macrophage density between P3 WT and $\mathrm{Lamc}^{-1-}$ central retinas (Supplemental Figure S1D). More important, unlike the central retina, no significant difference in microglial density between P3 WT and $L a m c 3^{-1-}$ peripheral retinas was observed (Figure 2, E and F). Taken together, these results indicate that in the absence of $\gamma 3$-containing laminins, microglial density is increased exclusively in the central retina around the growing vascular tree.

\section{Lamc3 $^{-/-}$SVP Exhibits Increased Microglial Association with the Vascular Branch Points}

Previous studies reported a direct correlation between the microglial number and vascular branching density in the CNS, suggesting that microglia facilitate vascular branching. ${ }^{10,12}$ Microglial density is positively correlated with vascular growth-when there is low vascular coverage, microglial density is low; as the vasculature develops, the microglial density increases specifically in association with the vasculature (Supplemental Figure S2). At P0, the WT retina is mostly avascular and the microglial density is low in both central and peripheral regions (Supplemental Figure S2A). At P3, the microglial density is increased around the newly vascularized central retina compared to the avascular peripheral retina (Supplemental Figure S2B). By $\mathrm{P} 8$, as the whole of the retina is vascularized, the microglial density is equally high in both central and peripheral regions

\footnotetext{
Figure $3 \mathrm{LamC}^{-/-}$SVP exhibits increased microglial association with the vascular branch points. A: Schematic of the retinal region (in the presence of mature astrocyte template and SVP). Images in B were acquired from the boxed area. B: P3 WT and Lamc3 $^{-/}$- retinal whole mounts were stained with isolectin B4 (IB4; green) and anti-F4/80 (red) antibody. Central retinal images are displayed. Arrows point at microglial cells in association with the vascular branch points. C: Quantification of microglia in association with the vascular branch points in P3 WT and Lamc3 ${ }^{-/-}$retinas. D: P5 WT and Lamc3 ${ }^{-/-}$retinal whole mounts, treated as indicated, were stained with IB4 (white). Representative images of $\mathrm{IB}^{+}{ }^{+}$microglia are displayed. E: Quantification of mitotic microglia density in P5 WT and $\mathrm{LamC3}^{-/-}$retinas with indicated treatments. F: P5 WT and $\mathrm{LamC}^{-/-}$retinal whole mounts, treated as indicated, were stained with IB4 (green). Representative images of the whole vascular tree in one retinal quadrant are displayed. Insets: Vascular fronts and nascent plexus. G: Quantification of vascular branching density in P5 WT and Lamc $^{-/-}$retinas with indicated treatments. Data are expressed as means \pm SEM. $n=3(\mathbf{C}, \mathbf{E}$, and $\mathbf{G}) .{ }^{*} P<0.05$ versus WT; ${ }^{\dagger} P<0.05$ versuS Lamc $^{-1-}$. Scale bars: $32 \mu \mathrm{m}$ (B, D, and insets in F); $160 \mu \mathrm{m}(\mathbf{F})$. GCL, ganglion cell layer; $0 \mathrm{NH}$, optic nerve head.
} 
(Supplemental Figure S2C). These results suggest that retinal microglia are heavily recruited to the growing vascular tree, where they play a role in vascular patterning.

Because of both the increased microglial density and hyperbranching, we specifically assayed if there was an increase in microglial association with the vessel branch points in the $\mathrm{Lamc}^{-1-}$ retina. For this analysis, the number of microglia at the vascular branch points in nascent SVP were measured (Figure 3A). There was a significant increase in the number of branch point-associated microglia in P3 Lamc $3^{-1-}$ retinas compared to WT (Figure 3, B and C). These results support the hypothesis that the higher microglial density in the $\mathrm{Lamc}^{-1-}$ retina drives more vascular anastomotic events, generating a denser hyperbranched SVP.

To directly test that hypothesis, WT mice were treated with LPS (Supplemental Figure S3A). LPS treatment did not have any obvious effect on astrocyte density (Supplemental Figure S3B). However, microglial density was increased in LPS-treated WT retinas compared to the saline-treated controls (Figure 3D); an increase in proliferation of microglial cells in LPS-treated retinas was also seen (Figure 3E). There was a concomitant increase in vascular branching in LPS-treated WT retinas compared to saline-treated controls (Figure 3, F and G), which supports the hypothesis that microglial recruitment is driving vascular branching. In the reciprocal experiment, $L a m c 3^{-l-}$ mice were treated with minocycline (Supplemental Figure S3A), to block microglial activation. Like LPS treatment, minocycline treatment also did not affect astrocyte density (Supplemental Figure S3C). However, minocycline treatment of $\mathrm{Lamc}^{-/-}$retinas reduced microglial density compared to saline-treated controls (Figure 3D); at the same time, it reduced microglial proliferation (Figure 3E). The net result was that vascular branching density decreased in minocycline-treated $\mathrm{Lamc}^{-{ }^{-1}}$ retinas to the WT level (Figure 3, F and G); minocycline was able to block the $2 a m c 3^{-l-}$ phenotype. Minocycline treatment of WT retinas did not alter either microglial density or vascular branching density when compared to the saline-treated control (Supplemental Figure S3D).

Taken together, these data further support the hypothesis that microglial density modulates vascular branching density. Specifically, the higher microglial density in the Lamc $3^{-/-}$retina drives the hyperbranching of the nascent SVP. More important, the data also raise the possibility that the vascular basement membrane may regulate the activation state of the microglia and thereby regulate vascular pattern.

\section{Interaction with Astrocyte-Derived ECM Influences Microglial Activation}

We performed a series of experiments designed to test the hypothesis that vascular basement membrane modulates the activation of microglial cells in the retina. First, we examined how microglial activation is regulated during developmental angiogenesis in the WT retina. As retinal SVP develops as a wave across the retina, at early postnatal ages, the development process is spatially arrayed across the retinal surface. Thus, we were able to analyze three discrete developmental compartments in the P3 WT retina: i) the central retina, which contains a mature astrocyte template and a well-established vascular tree, ii) the midperipheral retina, which has a mature astrocyte template but lies ahead of the vascular front, and iii) the peripheral retina, which lacks both a mature astrocyte template and developing vasculature (Figure $4 \mathrm{~A}$ ).

Both morphological and immunological assays were used to assess microglial activation. Classically, resting and activated microglia are distinguished by their morphology. Resting microglial cells have small cell bodies that are highly branched (ramified), whereas activated microglia have large amoeboid cell bodies. Using both F4/80 and lectin labeling, we observed that microglial cells exhibited mostly amoeboid (activated) morphology in central and midperipheral retinas (Figure 4B). However, microglia in the peripheral retina exhibited ramified (resting) morphology (Figure 4B). We also used CD68 to label activated microglia. CD68 ${ }^{+}$microglia were present only in central and midperipheral retinas (Figure 4B); this observation is consistent with activated, amoeboid morphology, observed in these regions. On the other hand, microglia in the peripheral retina did not exhibit CD68 immunoreactivity, consistent with the resting, ramified morphology they adopt in this region (Figure 4B).

As we observed that amoeboid, $\mathrm{CD}^{+} 8^{+}$(activated) microglia are present only around the mature astrocyte template and absent in the peripheral retina, we asked if astrocyte-microglia interaction is important for microglial activation. Indeed, we observed that $\mathrm{CD} 68^{+}$microglia are in close association with the mature astrocyte template in the P3 WT central retina (Figure 4C). Moreover, three-dimensional reconstruction of astrocyte-microglial pairs from the central retina further revealed that $\mathrm{CD} 68^{+}$ microglia are in contact with the mature astrocyte template (Figure 4D). No CD68 ${ }^{+}$microglia were seen in the absence of the mature astrocyte template (Figure 4C).

To directly test the hypothesis that contact with astrocytes modulates microglial activation, we developed an in vitro assay. Isolated astrocytes from WT mouse retinas were used as a source of cell-derived ECM. Astrocyte cultures that were $>95 \%$ pure (Supplemental Figure S4A) were grown for 4 days and assayed for laminin production. These astrocytes deposited a rich laminin matrix (Figure 4E), containing $\gamma 3$-chain (Supplemental Figure S4B). We decellularized these WT astrocyte cultures with dimethyl sulfoxide, leaving the astrocyte-derived ECM behind on the glass coverslips (Supplemental Figure S4B), and cultured WT rat brain-derived microglia on the WT astrocytederived ECM. Microglia cultured directly on glass coverslips were used as controls. Microglial cells cultured on WT astrocyte-derived ECM displayed amoeboid (activated) 
A

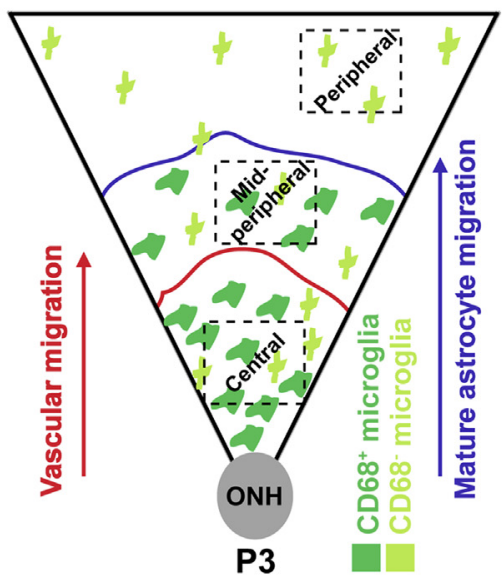

B
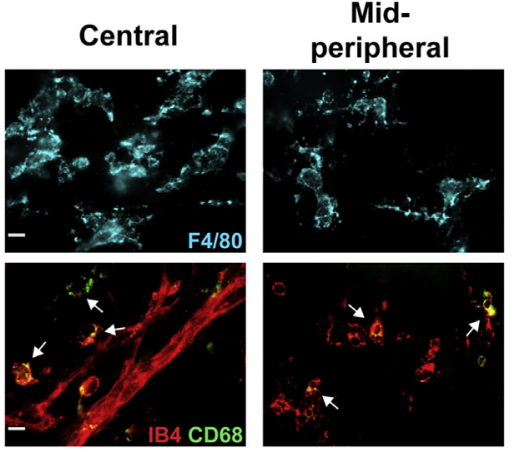

P3 WT
C

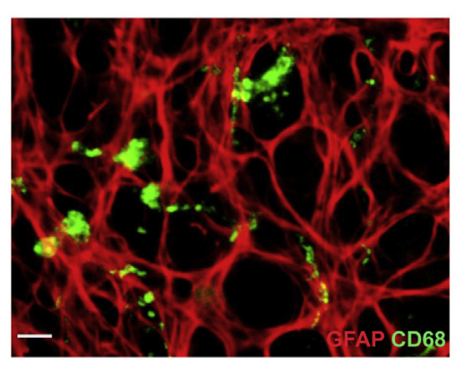

P3 WT
Peripheral

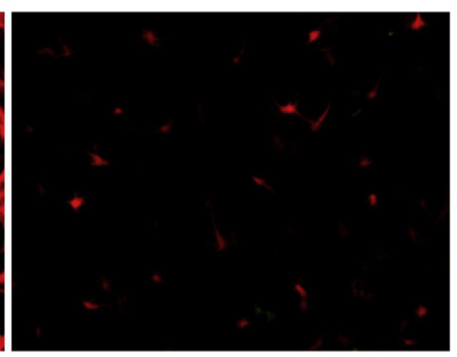

(n)

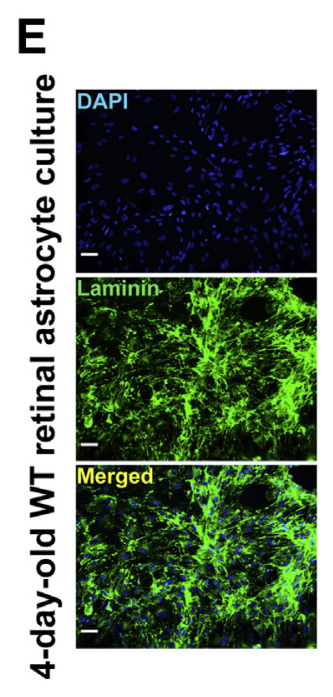

F

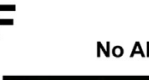

D

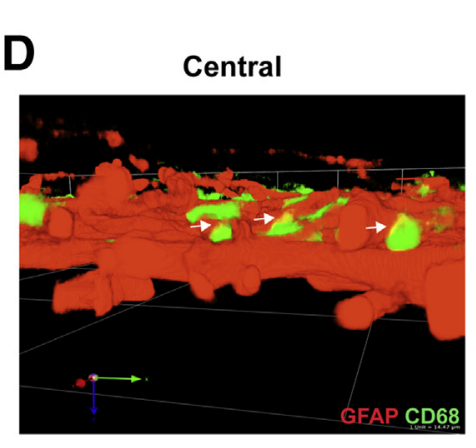

P3 WT
Peripheral

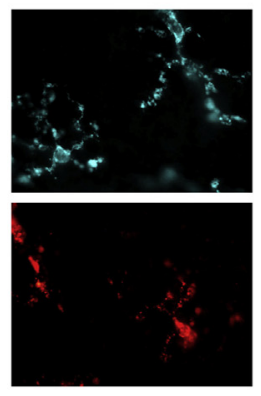

8

\section{G}

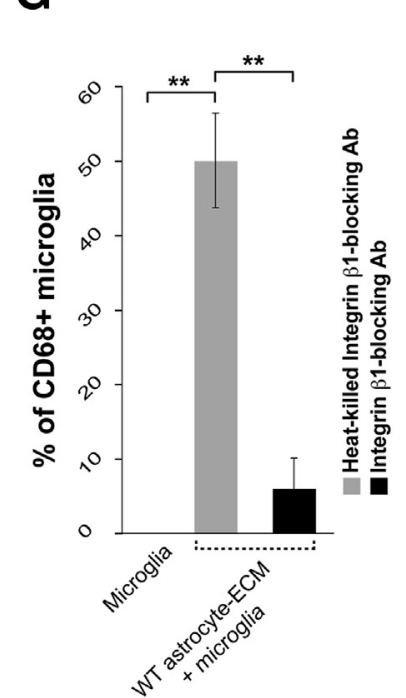

Figure 4 Interaction with the astrocyte-derived extracellular matrix (ECM) influences microglial activation. A: Schematic of the retinal regions (central, midperipheral, and peripheral). Images in B were acquired from boxed areas. B: P3 WT retinal whole mounts were stained with either anti-F4/80 (cyan) antibody or isolectin B4 (IB4; red) and anti-CD68 (green) antibody. White arrows point at CD68 ${ }^{+}$microglial cells in central and midperipheral retinas. C: P3 WT retinal whole mounts were stained with anti-CD68 (green) and anti-glial fibrillary acidic protein (GFAP; red) antibodies. Representative images of central and peripheral retinas are displayed. D: Representative three-dimensional reconstruction of the image from the whole-mounted P3 WT central retina stained with anti-CD68 (green) and anti-GFAP (red) antibodies. White arrows indicate contacts between $\mathrm{CD}^{+} 8^{+}$microglial cells and GFAP ${ }^{+}$(mature) astrocyte template; green arrow, $x$ axis; red arrow, $y$ axis; and blue arrow, $z$ axis. E: WT retinal astrocytes grown on glass coverslips for 4 days were stained with anti-pan-laminin (green) antibody and nuclear marker DAPI (blue). F: WT rat brain microglial cells, grown under indicated conditions, were stained with IB4 (green) and anti-CD68 (white) antibody. Yellow arrows indicate $\mathrm{CD}^{+} 8^{+}$(activated) microglial cells. G: Quantification of the percentage of CD68 ${ }^{+}$(activated) microglia grown under indicated conditions. Data are expressed as means \pm SEM. $n=3(\mathbf{G})$. ${ }^{*} P<0.01$. Scale bars: $11 \mu \mathrm{m}(\mathbf{B}) ; 16 \mu \mathrm{m}(\mathbf{C}) ; 60 \mu \mathrm{m}(\mathbf{E}) ; 32 \mu \mathrm{m}$ (F). Ab, antibody; $0 \mathrm{NH}$, optic nerve head. 

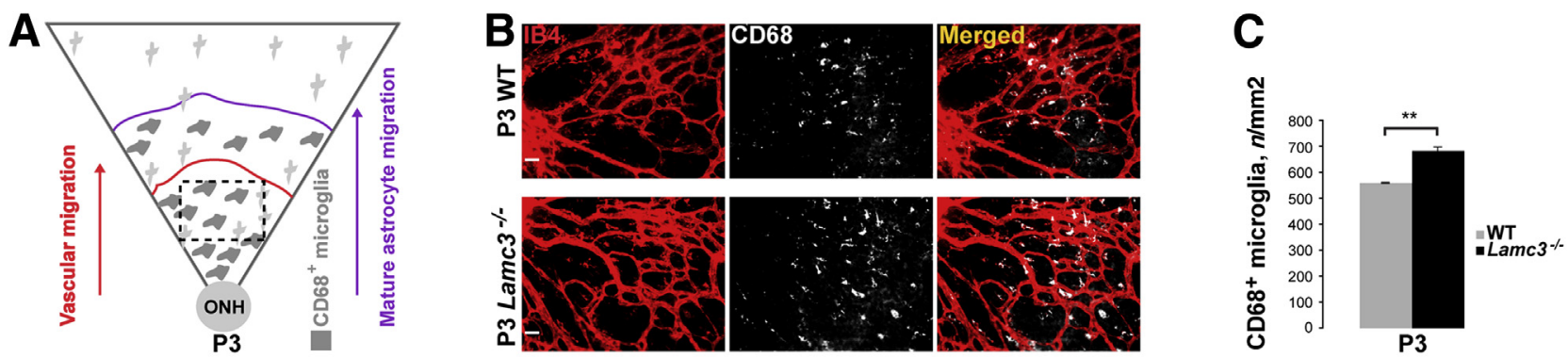

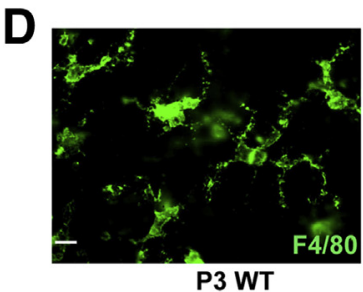

G

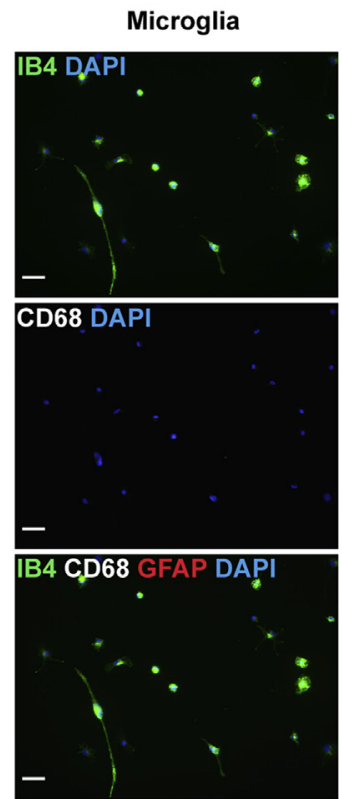

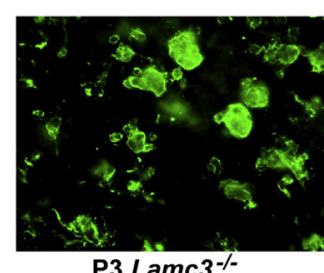

P3 Lamc3 $^{-/-}$

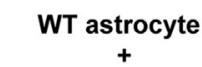

microglia
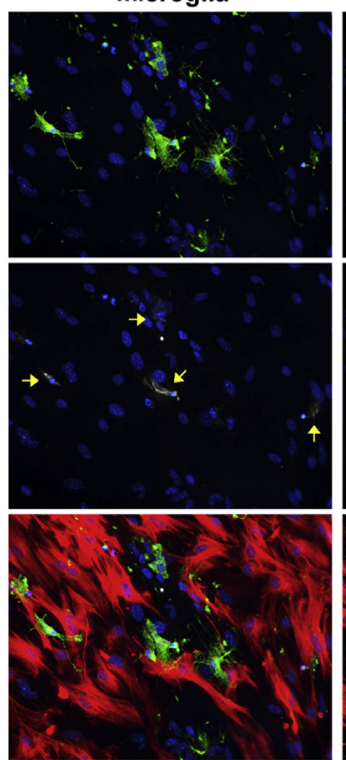

E

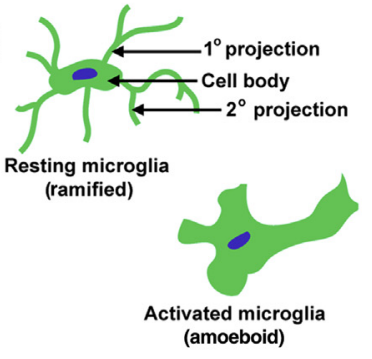

Lamc $3^{-/-}$astrocyte

$+$

Microglia

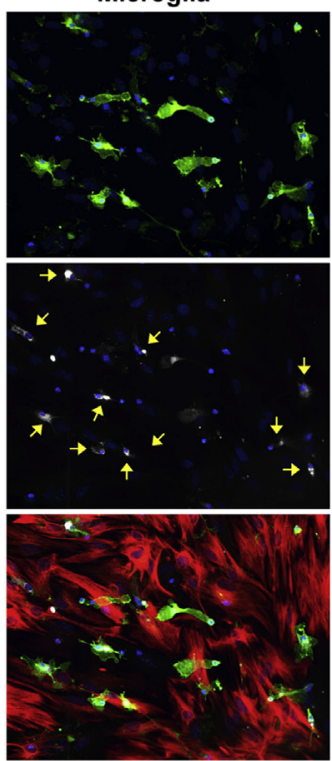

$\mathbf{F}$

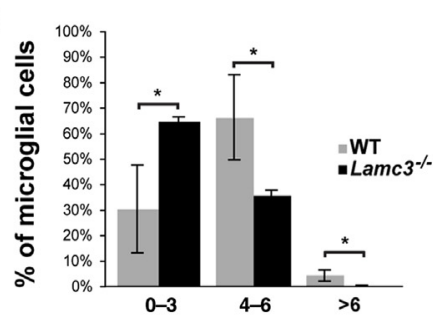

H

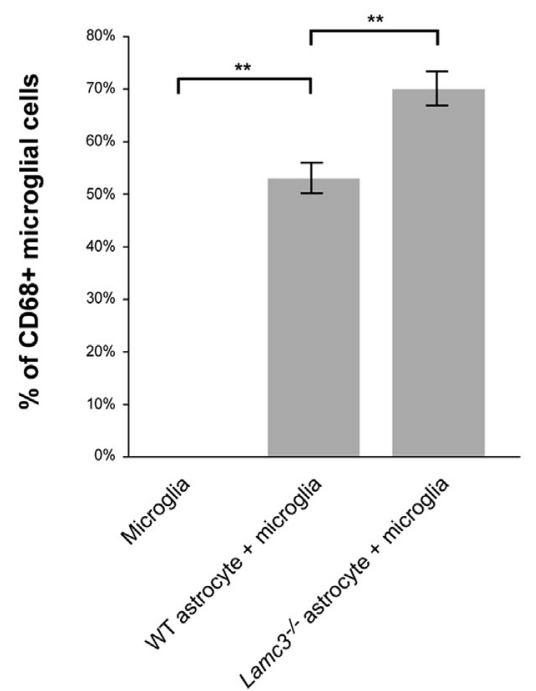

Figure 5 Microglia are hyperactivated around the $\mathrm{Lamc}^{-1-}$ retinal SVP. A: Schematic of the central retinal region (in the presence of mature astrocyte template and SVP). Images in B were acquired from the boxed area. B: P3 WT and $L a m 3^{-1-}$ retinal whole mounts were stained with isolectin B4 (IB4; red) and anti-CD68 (white) antibody. C: Quantification of the CD68 ${ }^{+}$(activated) microglia density in P3 WT and $\mathrm{Lamc}^{-/-}$central retinas. D: P3 WT and Lamc3 ${ }^{-/-}$retinal whole mounts were stained with anti-F4/80 (green) antibody. E: Pictorial depictions of typical morphologies of the resting and activated microglial cells. F: Quantification of the microglial cells with indicated number of primary projections/cell in P3 WT and Lamc3 ${ }^{-1-}$ central retinas. G: WT rat brain microglial cells, either grown alone or co-cultured with astrocytes of indicated genotypes, were stained with IB4 (green), anti-CD68 (white) antibody, and DAPI (blue). Arrows point at $\mathrm{CD}^{+} 8^{+}$(activated) microglial cells. H: Quantification of the percentage of $\mathrm{CD}^{+} 8^{+}$(activated) microglia grown under indicated conditions. Data are expressed as means \pm SEM. $n=3$ (C, F, and $\mathbf{H}) .{ }^{*} P<0.05,{ }^{* *} P<0.01$. Scale bars: $32 \mu \mathrm{m}$ (B); $16 \mu \mathrm{m}$ (D); $60 \mu \mathrm{m}(\mathbf{G})$. 0NH, optic nerve head.

morphology (Supplemental Figure S4C), and 50\% of the microglial cells cultured on WT astrocyte-derived ECM were $\mathrm{CD}^{+} 8^{+}$(Figure 4, F and G). Control microglial cells had small cell bodies and thin processes (Supplemental Figure S4C) and were CD68 ${ }^{-}$(Figure 4, F and G). A previous report demonstrated that microglia bind to the laminins via integrin $\alpha 6 \beta 1 .^{15}$ Thus, we blocked integrin signaling in the in vitro assay. Blocking integrin $\beta 1-$ mediated signaling significantly reduced the percentage of $\mathrm{CD} 8^{+}$microglia grown on WT astrocyte-derived ECM (Figure 4, F and $\mathrm{G}$ ); microglia under these conditions also had resting morphology (small cell bodies and thin processes) (Supplemental Figure S4C). Taken together, these results suggest that the interaction with astrocytederived ECM is able to activate microglia via integrin $\beta 1-$ mediated signaling. 


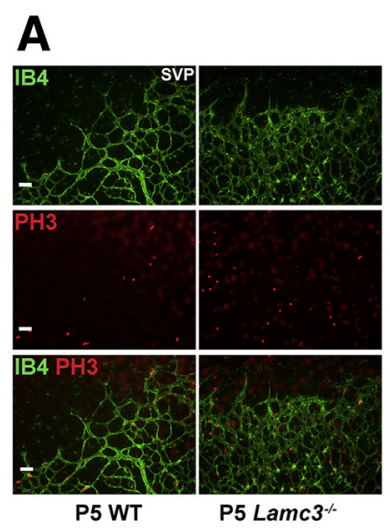

\section{B}

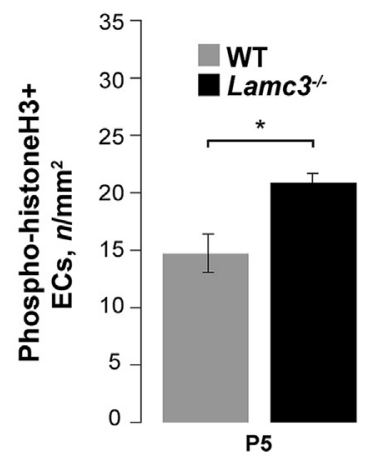

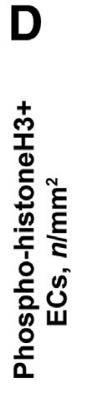

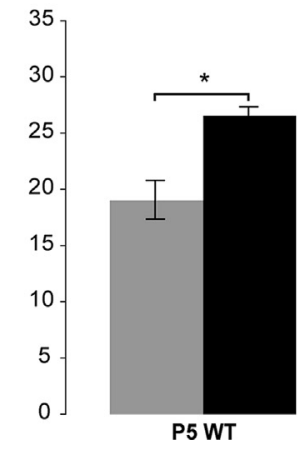

C
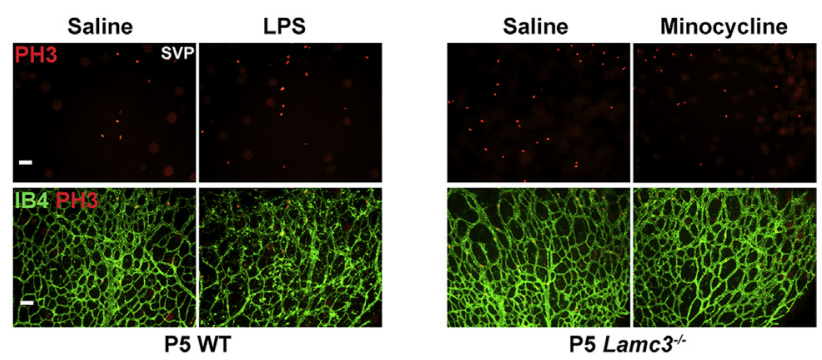

Figure 6 Microglial activation state affects endothelial cell proliferation. A: P5 WT and $\mathrm{LamC}^{-/-}$retinal whole mounts were stained with anti-phospho-histone H3 (PH3; red) antibody and isolectin B4 (IB4; green). B: Quantification of mitotic endothelial cell (EC) density in P5 WT and $\mathrm{LamC}^{-1-}$ retinas. C: P5 WT and $\mathrm{LamC}^{-1-}$ retinal whole mounts, treated as indicated, were stained with IB4 (green) and anti-PH3 (red) antibody. D: Quantification of mitotic endothelial cells in the P5 WT and $\mathrm{LamC}^{-1-}$ retinas with indicated treatments. Data are expressed as means \pm SEM. $n=3$ (B and $\mathbf{D}) .{ }^{*} P<0.05$ versus WT; ${ }^{\dagger} P<0.05$ versus Lamc $^{-/-}$. Scale bars $=60 \mu \mathrm{m}(\mathbf{A}$ and $\mathbf{C})$.

\section{Microglia Are Hyperactivated around the $\mathrm{Lamc}^{-/-}$ SVP}

A previous study demonstrated that the presence of laminin $\gamma 3$-chain inhibits laminins from binding to integrin receptors. ${ }^{21}$ Logically, the genetic deletion of laminin $\gamma 3$-chain should up-regulate integrin $\beta 1$-mediated signaling. Given our data above, we hypothesized that an up-regulation of integrin $\beta 1-$ mediated signaling would result in hyperactivation of microglia in the $\operatorname{Lamc}^{-1-}$ retina. To test this hypothesis, we compared microglial activation between WT and $L a m c 3^{-1-}$ central retinas at P3 (Figure 5A). There were significantly more $\mathrm{CD}^{+} 8^{+}$(activated) microglia in $\mathrm{Lamc}^{-1-}$ central retinas than the WT (Figure 5, B and C). Moreover, significantly more microglial cells had activated (amoeboid) morphology with fewer primary processes in $\mathrm{Lamc}^{-1-}$ central retinas than in WT (Figure 5, D-F). Together, these results support the hypothesis that microglia are hyperactivated around the $\mathrm{Lamc}^{-/-}$SVP.

Next, we asked whether the hyperactivation of microglia is because of altered astrocyte-microglia interactions in the Lamc $3^{-1-}$ retina. Astrocytes were isolated from both WT and $\mathrm{Lamc}^{-1-}$ retinas. WT rat brain-derived microglia were co-cultured with either WT or $L a m c 3^{-1-}$ retinal astrocytes. Monoculture of microglia was used as control.

None of the microglial cells cultured alone displayed CD68 immunoreactivity (Figure 5, G and H). On the other hand, approximately $50 \%$ of the microglial cells co-cultured with the WT astrocytes were $\mathrm{CD}^{+} 8^{+}$(Figure 5, G and H), similar to the percentage we obtained with microglia grown on the WT astrocyte-derived ECM (Figure 4, F and G). Finally, the percentage of $\mathrm{CD}^{+} 8^{+}$(activated) microglia was significantly higher when co-cultured with the $\mathrm{Lamc}^{-1-}$ astrocytes (Figure 5, G and $\mathrm{H}$ ), consistent with the in vivo results. Thus, retinal microglia are hyperactivated in the Lamc $^{-1-}$ retina. Moreover, this hyperactivation of microglia in the $L a m c 3^{-1-}$ retina appears to be because of their interaction with the mutant astrocyte-derived ECM.

\section{Microglial Activation State Affects Endothelial Cell Proliferation}

Activated microglia secrete proangiogenic factors. ${ }^{14}$ Because microglia around the growing SVP are hyperactivated in the Lamc $^{-1-}$ retina (Figure 5, B-F), we next assayed endothelial cell proliferation and demonstrated that there were significantly more mitotic endothelial cells in P5 $\mathrm{Lamc}^{-1-}$ retinas compared to the WT controls (Figure 6, A and B), consistent with microglial hyperactivation of the $L a m c 3^{-1-}$ retina.

To establish a causal link between microglial activation and endothelial proliferation, we examined the effects of pharmacological manipulations of microglial activation on endothelial cell proliferation. First, we showed that pharmacological activation of microglia by LPS treatment in WT retinas led to increased endothelial cell proliferation (Figure 6, C and D), compared to the saline-treated controls. Thus, activation of microglia was sufficient to phenocopy Lamc3 deletion with respect to endothelial proliferation. Reciprocally, pharmacological blockade of microglial activation by minocycline treatment of $\mathrm{Lamc}^{-1-}$ retinas 

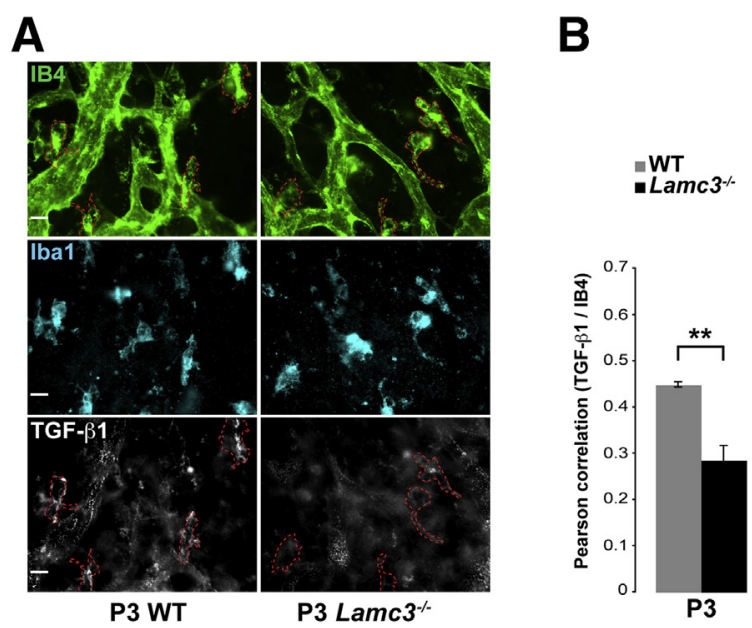

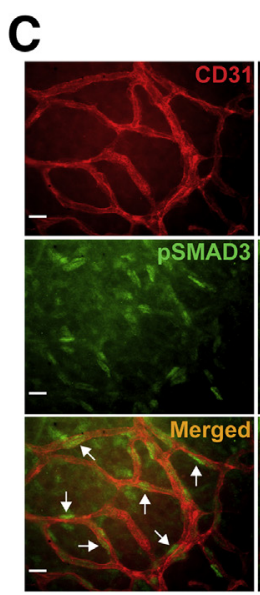

P3 WT

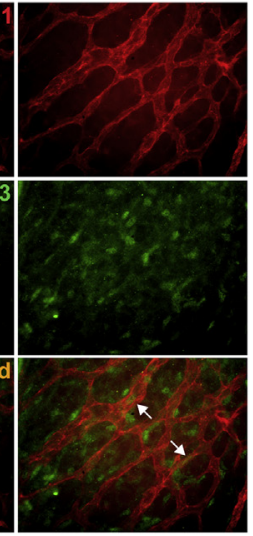

P3 Lamc $3^{-1-}$
D

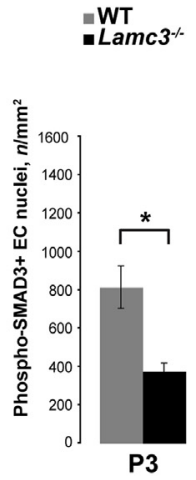

Figure 7 Microglial transforming growth factor (TGF)- $\beta 1$ expression and endothelial TGF- $\beta$ pathway are altered at the SVP of the Lamc3 ${ }^{-/-}$retina. A: P3 WT and $\mathrm{LamC}^{-1-}$ retinal whole mounts were stained with isolectin B4 (IB4; green), anti-Iba1 (cyan), and anti-TGF- $\beta 1$ (white) antibodies. Representative images around the SVP are displayed. Red dashed lines outline representative microglial cells, corroborating with Iba1 labeling. B: Quantification of microglial TGF- $\beta 1$ expression in the P3 WT and $\mathrm{Lamc}^{-/-}$retinas. C: P3 WT and $\mathrm{Lamc}^{-1-}$ retinal whole mounts were stained with anti-CD31 (red) and anti-phospho-SMAD3 (pSMAD3; green) antibodies. Representative images around the SVP are displayed. Arrows point at representative pSMAD3 ${ }^{+}$endothelial cells. D: Quantification of $\mathrm{PSMAD}^{+}$endothelial cell (EC) density in P3 WT and $\mathrm{Lamc}^{-/-}$retinas. Data are expressed as means \pm SEM. $n=3(\mathbf{B}$ and $\mathbf{D}) .{ }^{*} P<0.05,{ }^{* *} P<0.01$. Scale bars: $12 \mu \mathrm{m}(\mathbf{A}) ; 16 \mu \mathrm{m}(\mathbf{C})$.

decreased endothelial cell proliferation compared to salinetreated controls (Figure 6, C and D). Together, these results are consistent with the hypothesis that increased activation of microglia in the $L a m c 3^{-1-}$ retina is likely responsible for the hyperproliferative SVP.

\section{Microglial TGF- $\beta 1$ Expression and Endothelial TGF- $\beta$ Pathway Are Affected in the $\mathrm{LamC}^{-/-}$Retina}

A previous study reported that microglia-derived TGF- $\beta 1$ inhibits endothelial cell proliferation. ${ }^{14}$ Because we observed hyperproliferation of endothelial cells in the Lamc $^{-/-}$SVP, we analyzed TGF- $\beta 1$ expression in the SVP-associated microglia in the Lamc $^{-1-}$ retina. TGF- $\beta 1$ expression was significantly down-regulated in the microglial cells around the $\mathrm{Lamc}^{-1-}$ SVP (Figure 7, A and B). Not only was TGF- $\beta 1$ expression in microglial cells down-regulated, but its down-stream signaling cascade in endothelial cells was affected as well. Specifically, the density of phospho-SMAD3 ${ }^{+}$endothelial nuclei was significantly reduced in $L a m c 3^{-1-}$ retinas than that in the WT (Figure 7, C and D). Together, these results strongly link the changes in microglial TGF- $\beta 1$ expression with endothelial cell proliferation in the $L a m c 3^{-1-}$ retina.

\section{Pharmacological Blocking of TGF- $\beta$ Signaling Increases Endothelial Cell Proliferation, without Affecting Vascular Branching Density}

Our results suggest that increased microglial density in the Lamc $3^{-/-}$retina is responsible for higher vascular branching density. Moreover, microglial hyperactivation in the Lamc $3^{-1-}$ retina results in a down-regulation of microglial
TGF- $\beta 1$ expression and increased endothelial cell proliferation. Although alterations in vascular branching and endothelial cell proliferation are spatially and temporally linked in the $\mathrm{Lamc}^{-/-}$retina, they may be separable developmental events. If they are separable, pharmacological blockade of the TGF- $\beta$ signaling pathway should logically alter endothelial cell proliferation, and leave vascular branching density unaffected as long as microglial density is not disrupted.

To test this hypothesis, we i.p. injected P3 WT mice with a specific inhibitor of the TGF- $\beta$ receptor I, SB- $431542^{22}$ (Figure 8A). There was no obvious difference in microglial density between saline-treated and SB-431542-treated WT retinas at P5 (Supplemental Figure S5). Moreover, vascular branching density was also unaffected in SB431542-treated retinas at P5 (Figure 8, B and C). On the other hand, endothelial cell proliferation was significantly increased in SB-431542-treated retinas at P5 (Figure 8, D and E). Thus, blocking TGF- $\beta$ signaling reproduced only one aspect of the $\operatorname{Lamc}^{-/-}$phenotype-the hyperproliferation of endothelial cells.

\section{Different Laminin Isoforms Have Different Effects on Microglial TGF- $\beta 1$ Expression and Endothelial Cell Proliferation}

Finally, we asked whether different laminin isoforms vary in their effects on microglial TGF- $\beta 1$ expression and endothelial cell proliferation. To address that question, we analyzed activated microglial phenotype around laminin $\beta 2$-null $\left(\right.$ Lamb2 $\left.^{-/-}\right)$retinal SVP. There was no significant difference in activated microglial density between WT and $\mathrm{Lamb2}^{-/-}$retinas (Supplemental Figure S6A). Consistent 

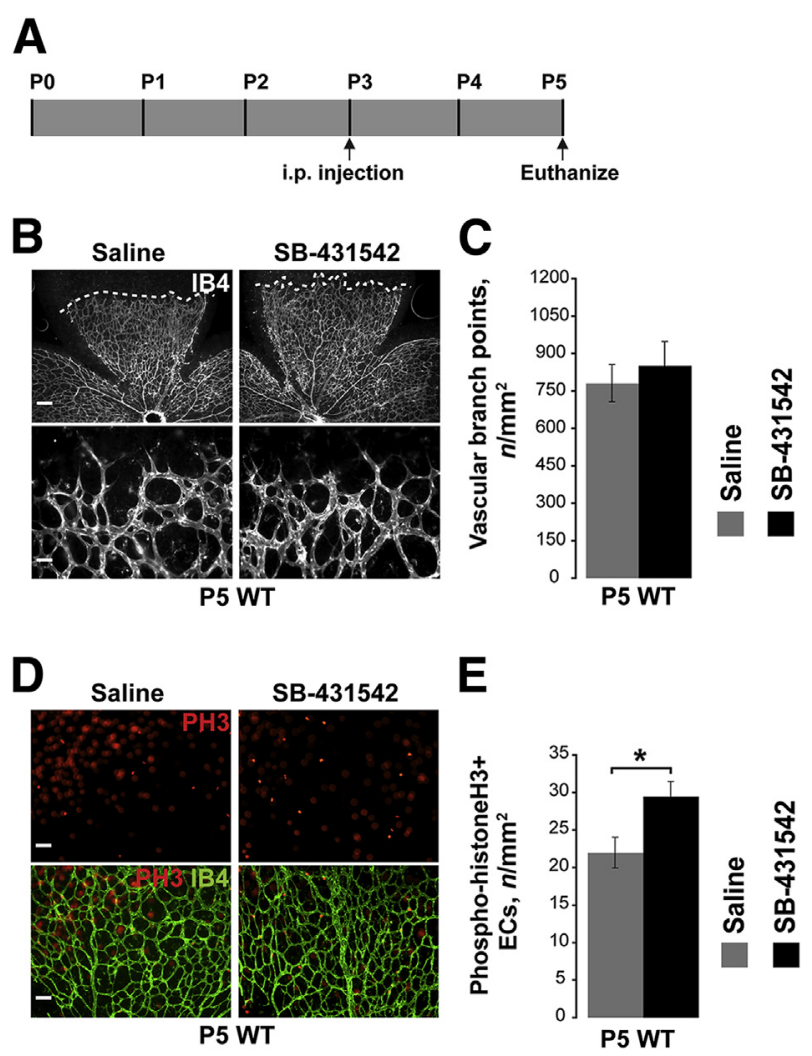

Figure 8 Pharmacological blocking of transforming growth factor- $\beta$ signaling increases endothelial cell proliferation, but not vascular branching density. A: Experimental paradigm of i.p. SB-431542 injection. B: P5 WT retinal whole mounts, treated as indicated, were stained with isolectin B4 (IB4; white). Top row: Lower magnification. Bottom row: Higher magnification. White dashed lines indicate the extent of vascular tree migration. C: Quantification of vascular branching density in P5 WT retinas with indicated treatments. D: P5 WT retinal whole mounts, treated as indicated, were stained with IB4 (green) and anti-phospho-histone H3 ( $\mathrm{PH} 3$; red) antibody. E: Quantification of mitotic endothelial cell (EC) density in P5 WT retinas with indicated treatments. Data are expressed as means \pm SEM. $n=3$ (C and E). ${ }^{*} P<0.05$. Scale bars: $160 \mu \mathrm{m}$ (B, top row); $32 \mu \mathrm{m}$ (B, bottom row); $60 \mu \mathrm{m}$ (D).

with the in vivo observation, co-culture with $L a m b 2^{-/-}$ astrocytes did not significantly alter microglial activation in vitro (Supplemental Figure S6B). However, microglial TGF- $\beta 1$ expression was significantly higher in Lamb2 $2^{-1-}$ retinas compared to the WT (Figure 9, A and B). Consistently, phosho-SMAD3 ${ }^{+}$endothelial cell density was also significantly higher in the $\mathrm{Lamb2}^{-/-}$retinal SVP (Figure 9, $\mathrm{C}$ and D). Consequently, there were significantly fewer mitotic endothelial cells in the $L a m b 2^{-1-}$ retinal SVP compared to the WT (Figure 9, E and F).

These results argue that different laminin isoforms vary in their effects on microglial TGF- $\beta 1$ expression, and consequently endothelial cell proliferation. Specifically, these results suggest that $\gamma 3$ - and $\beta 2$-containing laminins have opposite effects on microglial TGF- $\beta 1$ expression and endothelial cell proliferation. $\gamma 3$-Containing laminins inhibit endothelial cell proliferation by inducing microglial TGF- $\beta 1$ expression, whereas $\beta 2$-containing laminins induce endothelial cell proliferation by down-regulating microglial TGF- $\beta 1$ expression.

\section{Discussion}

Laminins Regulate Astrocyte Migration and Microglial Recruitment

Laminins containing the $\gamma 3$-chain are expressed in the capillary bed throughout the CNS, including the retina. ${ }^{19}$ This study, focused exclusively on the retina, has the dual goal of understanding the role of $\gamma 3$-containing laminins in the mammalian vascular basement membrane as well as elucidating some of the regulatory steps in retinal vascular development. Proper vascular development in the murine retina depends on the cross talk between multiple cell types. Radial migration of astrocytes over the surface of the retina forms a template that is critical for the expansion of the vascular tree. ${ }^{4,5}$ Simultaneously, retinal microglia play an important role in patterning the expanding vascular plexus, by mediating vascular anastomosis ${ }^{10,12}$ and endothelial cell proliferation. ${ }^{14}$ Studies from our laboratory and other groups have demonstrated that the laminins in the inner limiting membrane regulate astrocyte migration, and consequent vascular tree expansion. ${ }^{4,5}$ Specifically, we demonstrated that deletion of the Lamb2 gene causes a profound failure in astrocyte migration, whereas Lamc3 gene deletion slows astrocyte migration for several days. ${ }^{5}$ The deletion of the Lamal gene has effects similar to those of Lamb2. ${ }^{4}$ In all of these laminin mutants, subsequent vascular tree expansion is disrupted, albeit with different degrees of severity. In the Lama1 $^{-1-}$ and Lamb2 $2^{-1-}$ animals, SVP is profoundly disrupted and hyaloid vessels persist. ${ }^{4,5}$ However, in the Lamc $3^{-1-}$ animals, retinal SVP expands more slowly and appeared more highly branched than the WT. ${ }^{5}$

Our initial focus was on the role of laminins in regulating glial behavior. We considered the possibility that an increased astrocyte density might account for an increased vascular branching in the $\mathrm{Lamc}^{-/-}$retina. However, there was no significant difference in astrocyte density around the Lamc $^{-1-}$ retinal SVP compared to the WT, despite the slowed rate of astrocyte migration in these animals. ${ }^{5}$ Thus, unlike either the $\alpha 1$ - or $\beta 2$-containing laminins, $\gamma 3$ containing laminins have no role in patterning of the astrocyte template for SVP branching.

In contrast, we confirmed, quantitatively, an increased recruitment of microglial cells to the hyperbranched nascent SVP in the $L a m c 3^{-1-}$ retina. Previous studies reported that there is a positive correlation between the number of microglia and vascular branching density in the CNS, suggesting that microglia facilitate vascular branching in the CNS. ${ }^{0,12}$ However, what regulates microglial density around the developing vascular plexus is largely unknown. The absence of laminin $\gamma 3$-chain caused an increased microglial density specifically around the developing SVP. We confirmed that these cells were microglial cells (ie, not 


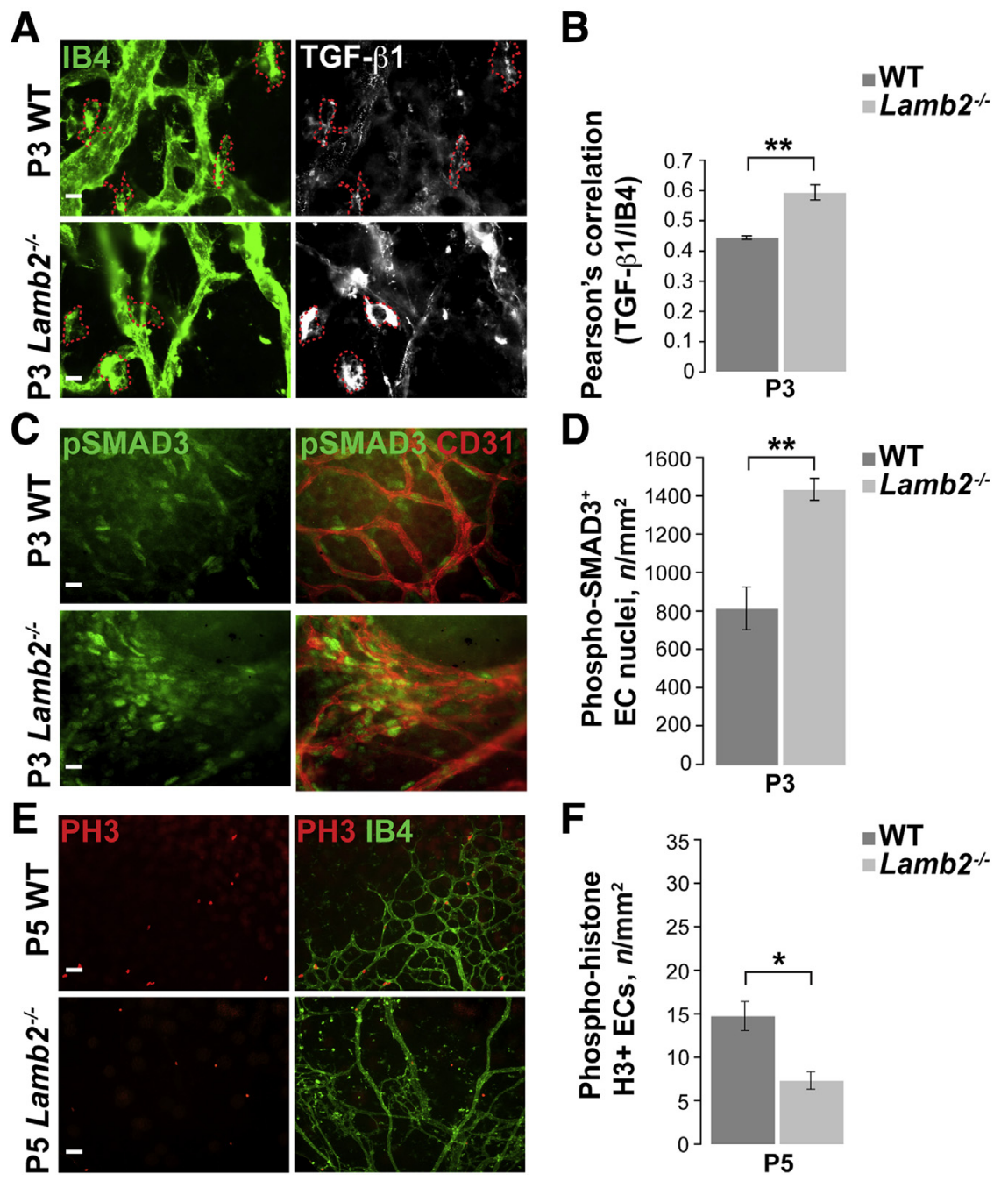

Figure 9 Lamb2 $^{-/-}$retinas exhibit upregulated microglial transforming growth factor (TGF)- $\beta 1$ expression and decreased endothelial cell proliferation. A: P3 WT and $\mathrm{Lamb2}^{-1-}$ retinal whole mounts were stained with isolectin B4 (IB4; green) and anti-TGF- $\beta 1$ (white) antibodies. Representative images around the SVP are displayed. Red dashed lines outline representative microglial cells. B: Quantification of microglial TGF- $\beta 1$ expression in P3 WT and $L a m b 2^{-/-}$retinas. C: P3 WT and $\mathrm{Lamb2}^{-/-}$retinal whole mounts were stained with anti-phospho-SMAD3 (pSMAD3; green) and anti-CD31 (white) antibodies. D: Quantification of $\mathrm{pSMAD3}^{+}$endothelial cell (EC) density in P3 WT and $\mathrm{Lamb2}^{-1-}$ retinas. E: P5 WT and $\mathrm{Lamb2}^{-1-}$ retinal whole mounts were stained with anti-phospho-histone $\mathrm{H} 3$ (PH3; red) antibody and IB4 (green). F: Quantification of mitotic endothelial cell density in P5 WT and Lamb2 ${ }^{-/-}$ retinal SVPs. Data are expressed as means \pm SEM (). $n=3$ (B, D, and F). ${ }^{*} P<0.05,{ }^{*} P<0.01$. Scale bars: $12 \mu \mathrm{m}(\mathbf{A}) ; 16 \mu \mathrm{m}(\mathbf{C}) ; 60 \mu \mathrm{m}(\mathbf{E})$.

invading perivascular macrophages) as there was no obvious difference in $\mathrm{CD} 45^{\text {high }}$ perivascular macrophage density between WT and Lamc $3^{-/-}$retinas. In addition, there were significantly more microglia closely associated with the vascular branch points in the $L a m c 3^{-1-}$ retinal SVP than in WT. Thus, the higher microglial density in the Lamc $^{-1-}$ retina appears to cause the increase in anastomotic events, resulting in a hyperbranched SVP. LPS treatment nonspecifically increased microglial density in WT retinas, which was accompanied by an increased vascular branching density, thereby generating an $\mathrm{Lamc}^{-1}$ - -like retinal vascular phenotype. Logically, pharmacological inhibition of microglial recruitment should reduce branching in the $L a m c 3^{-1-}$ retina. This is precisely the result achieved by treating early postnatal mice with minocycline (ie, the restoration of a WT vascular branching density in the $L a m c 3^{-1-}$ retina). These results strongly support our hypothesis that the higher microglial density in the $L a m c 3^{-1-}$ retina is responsible for generating a hyperbranched SVP. There are limitations to the specificity of these pharmacological manipulations. It is difficult to attribute the changes in vascular phenotype by these treatments solely to microglial physiology as LPS and minocycline treatment could affect multiple cell types. LPS treatment $^{23-25}$ and minocycline treatment ${ }^{26-28}$ are widely used to induce and block microglial activation in the CNS, respectively. However, neither LPS nor minocycline treatment had obvious effects on retinal astrocyte density, nor did we observe gross changes in the neuronal organization of these treated retinas. Thus, it appears that the changes in vascular phenotype observed with LPS and minocycline treatments are most likely microglia mediated.

Together, these results support the interpretation that the higher microglial density in the Lamc $^{-1-}$ retina is responsible for generating a hyperbranched SVP. Moreover, we also conclude that the composition of the vascular basement membrane is important in recruitment of microglia to the mural interface.

\section{ECM Composition Regulates Microglial Activation}

The results of this study also demonstrate that the vascular basement membrane components have a strong modulatory effect on the activation state of the mural associated 
microglia. To date, little is known about the role of ECM components in microglial activation. Microglia interact with the astrocyte-derived matrix during migration. ${ }^{15}$ However, the physiological consequence of that interaction is not well understood. One study reported that microglia adopt activated morphology on fibronectin and vitronectin, but not on laminin substrate. ${ }^{29}$ However, whether different laminin isoforms can have different effects on microglial activation is not clear. Using well-established parameters for microglial activation, CD68 expression, ${ }^{30-33}$ and amoeboid morphology, we show that activated microglia are found exclusively associated with the mature astrocyte template in the developing retina. In a direct in vitro assay, microglia were $\mathrm{CD}^{-} 8^{-}$and had a resting morphology when grown directly on glass coverslips. On the other hand, microglia were $\mathrm{CD}^{+} 8^{+}$and exhibited amoeboid morphology (activated) when grown on astrocyte-derived ECM in vitro. Consistent with previous studies that showed that microglia and macrophages bind to the laminin via integrin $\alpha 6 \beta 1,{ }^{15,34}$ when we blocked integrin $\beta 1$-mediated signaling in these cultures, microglial activation was significantly decreased. Taken together, these results strongly support the idea that the interaction with astrocyte-derived ECM is able to activate microglia via integrin $\beta 1$-mediated signaling.
Moreover, they suggest that the composition and integrity of the vascular basement membrane is a major cue in regulating microglial physiology.

\section{Laminin Regulates Down-Stream Signaling Events Leading To Endothelial Cell Proliferation}

Previous studies have demonstrated a link between microglia activation and TGF- $\beta 1$ production, and that resting microglia-derived TGF- $\beta 1$ inhibits endothelial cell proliferation in vitro. ${ }^{14}$ TGF- $\beta 1$ deletion causes vascular defects, including increased vessel density and endothelial cell proliferation. ${ }^{35}$ The overt phenotype of the $\operatorname{Lamc} 3^{-/-}$retina is similar: hyperactivated microglia and reduced TGF- $\beta 1$ expression, and reduced SMAD3 signaling in the endothelial cells with a consequent increase in endothelial cell proliferation. Based on our results, we propose that microglial recruitment and activation are the critical steps that link vascular branching and endothelial proliferation. During retinal angiogenesis, the vascular tree expands, following the astrocyte template. As the microglia are recruited to the growing vascular plexus, they facilitate vascular branching. At the same time, microglia come in contact with the astrocyte-derived ECM in the vascular BM. The interaction
A

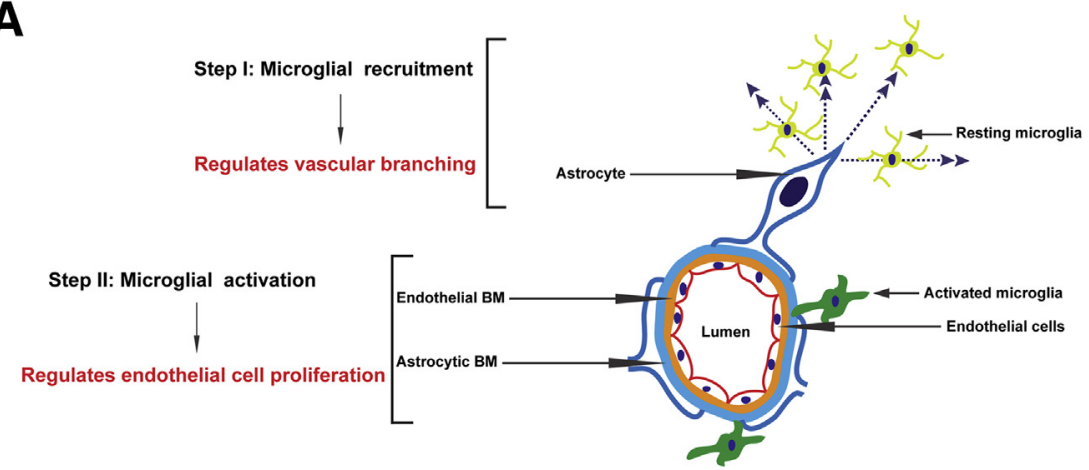

B

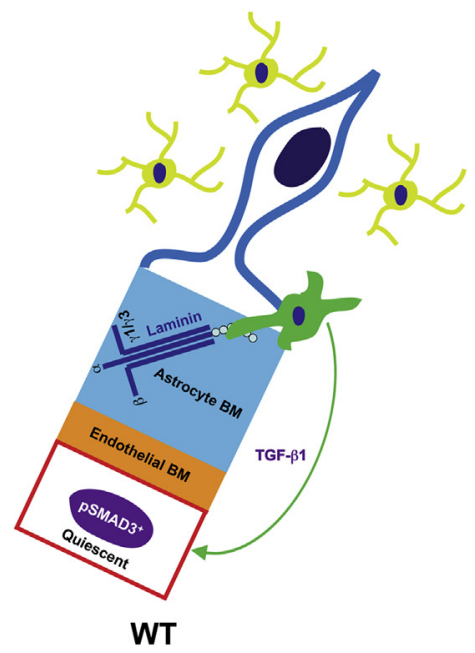

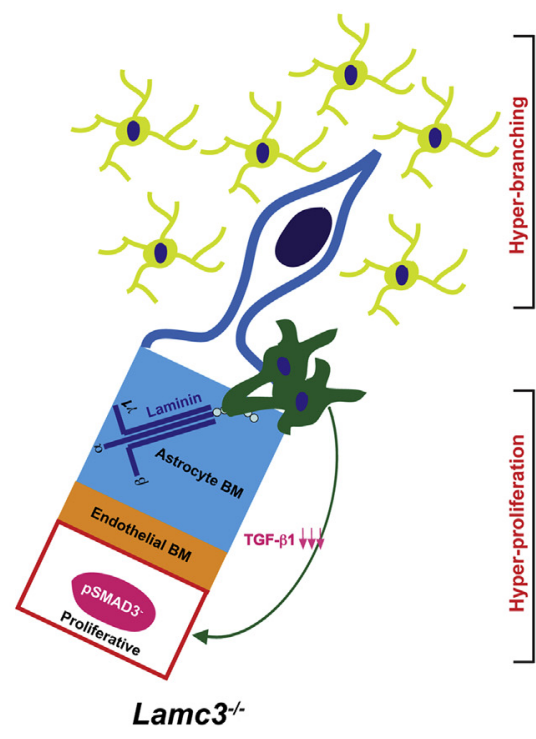

Figure 10 Laminin-dependent interaction between astrocytes and microglia regulates retinal angiogenesis. A: Vascular tree expands following the astrocyte template during retinal SVP development. As the microglia are recruited to the growing vascular tree, they facilitate vascular branching. At the same time, microglia come in contact with the astrocyte-derived extracellular matrix (ECM) in the vascular BM. The contact with astrocyte-derived ECM influences microglial activation. Microglial activation state influences endothelial cell proliferation. B: Microglia are recruited at a higher density to the developing $\mathrm{LamC}^{-/-}$retinal SVP. Higher microglial density likely causes hyperbranched SVP formation in the $\mathrm{LamC}^{-/-}$retina. In addition, the interaction with $\mathrm{LamC}^{-1-}$ astrocyte-derived ECM hyperactivates microglia. Hyperactivated microglia in the $\mathrm{LamC3}^{-/-}$retina drive increased proliferation of the endothelial cells because of down-regulated transforming growth factor (TGF)- $\beta$ signaling. pSMAD3, phospho-SMAD3. 
with astrocyte-derived ECM influences the microglial activation state (Figure 10A).

In the $\operatorname{Lamc}^{-1-}$ retina, microglia are recruited at a significantly higher density than the WT, which likely drives increased vascular branching. In addition, the interaction with $\mathrm{Lamc}^{-1-}$ astrocyte-derived ECM hyperactivates microglia, which likely drives increased proliferation of the endothelial cells because of down-regulated TGF- $\beta$ signaling (Figure 10B). Pharmacological inhibition of the TGF- $\beta$ pathway by SB-431542 treatment in the WT mice increased endothelial cell proliferation, but not vascular branching density. These data provide support to the model, as SB-431542 does not cross the blood-brain barrier ${ }^{36}$ and therefore its effects are logically restricted to the endothelial cells themselves. We cannot exclude the possibility that SB-431542 treatment could affect other mural cell types (smooth muscles or pericytes) either directly or indirectly via inhibited endothelial cells. Together, the weight of the data supports that microglia respond to the state of the gliaderived vascular basement membrane. The presence of $\gamma$ 3-laminins in the mural basement membrane prevents microglial activation and thereby keeps the microvessels stable. Down-regulation of the $\gamma 3$-laminin during pathobiologic events may then be the first step in the neovascular sprouting and remodeling that accompanies a myriad of vascular insults.

\section{Laminin-Mediated Regulation of Microglial Activation and Down-Stream Signaling Is Isoform-Specific}

Astrocytes make laminins containing $\beta 1^{3}-, \beta 2^{5}-, \gamma 1^{3}-$, and $\gamma 3$-chains. In the absence of laminin $\gamma 3$-chain, CD68 ${ }^{+}$ (activated) microglial density is significantly increased around the retinal SVP. Consistent with in vivo results, when microglia were co-cultured with $\mathrm{Lamc}^{-1-}$ retinal astrocytes, the percentage of $\mathrm{CD}^{+} 8^{+}$(activated) microglia was significantly higher than that of microglia-WT retinal astrocyte co-culture. In contrast, there was no change in microglial activation either in the Lamb2 $2^{-1-}$ retina in vivo or when microglia are co-cultured with $L a m b 2^{-1-}$ astrocytes. Consistent with this differential activation by $\gamma 3-$ and $\beta 2$-laminins, microglial TGF- $\beta 1$ expression and downstream SMAD3 signaling are also differentially regulated by laminin isoforms; microglial TGF- $\beta 1$ expression was down-regulated in the $\mathrm{Lamc}^{-1-}$ retina and up-regulated in the Lamb2 $^{-1-}$ retina. Consequently, phospho-SMAD3 ${ }^{+}$ endothelial cell density was increased, leading to reduced endothelial cell proliferation in the $L a m b 2^{-1-}$ retina. Thus, $\gamma 3$ - and $\beta 2$-containing laminins have opposite effects on microglial TGF- $\beta 1$ expression and endothelial cell proliferation; $\gamma 3$-containing laminins restrict endothelial cell proliferation by inducing microglial TGF- $\beta 1$ expression, whereas $\beta 2$-containing laminins induce endothelial cell proliferation by down-regulating microglial TGF- $\beta 1$ expression. Down-stream of microglial TGF- $\beta 1$ expression, $\gamma 3$-laminins activate endothelial SMAD3 signaling and inhibit endothelial cell proliferation, and thereby stabilize the microvessels, whereas $\beta 2$-laminins inhibit SMAD3 signaling and promote endothelial cell proliferation.

Our co-culture system is a mixed rat (microglial)-mouse (astrocytes) system. Under these circumstances, one might expect a high level of microglial activation. In our control cultures, $50 \%$ of the microglia are activated when cocultured with the WT astrocytes or on WT astrocyte-derived ECM. In contrast, growing microglia on $\mathrm{Lamc}^{-1-}$ retinal astrocytes increased activation significantly, whereas growing microglial on $\mathrm{Lamb2}^{-1-}$ did not. Thus, the coculture system is able to look, qualitatively, at microglial activation by glia-derived laminins.

Taken together, our study clearly shows that the $\gamma 3$-containing laminins play an important role in retinal angiogenesis by restricting vascular branching and endothelial proliferation during retinal angiogenesis. Lamc $^{-1-}$ retinas show characteristics of ischemic retinopathy, such as aberrant vascular branching and endothelial proliferation. Studies have shown that microglial activation plays an important role in disease prognosis of diabetic retinopathy ${ }^{37}$ and a mouse model of oxygen-induced retinopathy. ${ }^{38}$ The data presented herein suggest that laminins, in general, and $\gamma 3$-containing laminins, in particular, are key regulators of microglial activation during development and likely to play important roles in the pathobiology of retinal vasculature.

\section{Acknowledgments}

We thank Dr. Andrea Viczian (State University of New York Upstate Medical University), for providing SB431542 reagent.

W.J.B. is the guarantor of this work and, as such, had full access to all of the primary data in this study; W.J.B. and S.B. take responsibility for the integrity of the data and the accuracy of the analysis.

\section{Supplemental Data}

Supplemental material for this article can be found at http://dx.doi.org/10.1016/j.ajpath.2017.05.016.

\section{References}

1. Saint-Geniez M, D'Amore PA: Development and pathology of the hyaloid, choroidal and retinal vasculature. Int J Dev Biol 2004, 48: 1045-1058

2. Stahl A, Connor KM, Sapieha P, Chen J, Dennison RJ, Krah NM, Seaward MR, Willett KL, Aderman CM, Guerin KI, Hua J, Löfqvist C, Hellström A, Smith LE: The mouse retina as an angiogenesis model. Invest Ophthalmol Vis Sci 2010, 51:2813-2826

3. Sixt M, Engelhardt B, Pausch F, Hallmann R, Wendler O, Sorokin LM: Endothelial cell laminin isoforms, laminins 8 and 10 , play decisive roles in $\mathrm{T}$ cell recruitment across the blood-brain barrier in experimental autoimmune encephalomyelitis. J Cell Biol 2001, 153: 933-946 
4. Edwards MM, McLeod DS, Grebe R, Heng C, Lefebvre O, Lutty GA: Lama1 mutations lead to vitreoretinal blood vessel formation, persistence of fetal vasculature, and epiretinal membrane formation in mice. BMC Dev Biol 2011, 11:1-19

5. Gnanaguru G, Bachay G, Biswas S, Pinzón-Duarte G, Hunter DD, Brunken WJ: Laminins containing the $\beta 2$ and $\gamma 3$ chains regulate astrocyte migration and angiogenesis in the retina. Development 2013, 140:2050-2060

6. Aumailley M, Bruckner-Tuderman L, Carter WG, Deutzmann R, Edgar D, Ekblom P, Engel J, Engvall E, Hohenester E, Jones JC, Kleinman HK, Marinkovich MP, Martin GR, Mayer U, Meneguzzi G, Miner JH, Miyazaki K, Patarroyo M, Paulsson M, Quaranta V, Sanes JR, Sasaki T, Sekiguchi K, Sorokin LM, Talts JF, Tryggvason K, Uitto J, Virtanen I, von der Mark K, Wewer UM, Yamada Y, Yurchenco PD: A simplified laminin nomenclature. Matrix Biol 2005, 24:326-332

7. Macdonald PR, Lustig A, Steinmetz MO, Krammer RA: Laminin chain assembly is regulated by specific coiled-coil interactions. J Struct Biol 2010, 170:398-405

8. Colognato H, Yurchenco PD: Form and function: the laminin family of heterotrimers. Dev Dyn 2000, 218:213-234

9. Durbeej M: Laminins. Cell Tissue Res 2010, 339:259-268

10. Fantin A, Vieira JM, Gestri G, Denti L, Schwarz Q, Prykhozhij S, Peri F, Wilson SW, Ruhrberg C: Tissue macrophages act as cellular chaperones for vascular anastomosis down-stream of VEGF-mediated endothelial tip cell induction. Blood 2010, 116:829-840

11. Santos AM, Calvente R, Tassi M, Carrasco MC, Martín-Oliva D, Marín-Teva JL, Navascués J, Cuadros MA: Embryonic and postnatal development of microglial cells in the mouse retina. J Comp Neurol 2008, 2:224-239

12. Outtz HH, Tattersall IW, Kofler NM, Steinbach N, Kitajewski J: Notch1 controls macrophage recruitment and Notch signaling is activated at sites of endothelial cell anastomosis during retinal angiogenesis in mice. Blood 2011, 118:3436-3439

13. Stefater JAIII, Lewkowich I, Rao S, Mariggi G, Carpenter AC, Burr AR, Fan J, Ajima R, Molkentin JD, Williams BO, Wills-Karp M, Pollard J, Yamaguchi T, Ferrara N, Gerhardt H, Lang RA: Regulation of angiogenesis by a non-canonical Wnt-Flt1 pathway in myeloid cells. Nature 2011, 474:511-515

14. Welser JV, Li L, Milner R: Microglial activation state exerts a biphasic influence on brain endothelial cell proliferation by regulating the balance of TNF and TGF- $\beta 1$. J Neuroinflammation 2010, 7:1-7

15. Milner R, Campbell IL: Cytokines regulate microglial adhesion to laminin and astrocyte extracellular matrix via protein kinase Cdependent activation of the alpha6beta1 integrin. J Neurosci 2002, 22: $1562-1572$

16. Noakes PG, Gautam M, Mudd J, Sanes JR, Merlie JP: Aberrant differentiation of neuromuscular junctions in mice lacking s-laminin/laminin beta 2. Nature 1995, 374:258-262

17. Denes V, Witkovsky P, Koch M, Hunter DD, Pinzon-Duarte G, Brunken WJ: Laminin deficits induce alterations in the development of dopaminergic neurons in the mouse retina. Vis Neurosci 2007, 24:549-562

18. Pinzon-Duarte G, Daly G, Li YN, Koch M, Brunken WJ: Defective formation of the inner limiting membrane in laminin beta2- and gamma3-null mice produces retinal dysplasia. Invest Ophthalmol Vis Sci 2010, 51:1773-1782

19. Li YN, Radner S, French MM, Pinzon-Duarte G, Daly GH, Burgeson RE, Koch M, Brunken WJ: The $\gamma 3$ chain of laminin is widely but differentially expressed in murine basement membranes: expression and functional studies. Matrix Biol 2012, 31:120-134

20. Greter M, Merad M: Regulation of microglia development and homeostasis. Glia 2013, 61:121-127

21. Ido H, Ito S, Taniguchi Y, Hayashi M, Sato-Nishiuchi R, Sanzen N, Hayashi Y, Futaki S, Sekiguchi K: Laminin isoforms containing the $\gamma 3$ chain are unable to bind to integrins due to the absence of the glutamic acid residue conserved in the C-terminal regions of the $\gamma 1$ and $\gamma 2$ chains. J Biol Chem 2008, 283:28149-28157
22. Chen Y, Kam CS, Liu FQ, Liu Y, Lui VC, Lamb JR, Tam PK: LPSinduced up-regulation of TGF-beta receptor 1 is associated with TNFalpha expression in human monocyte-derived macrophages. J Leukoc Biol 2008, 83:1165-1173

23. Chen Z, Jalabi W, Shpargel KB, Farabaugh KT, Dutta R, Yin X, Kidd GJ, Bergmann CC, Stohlman SA, Trapp BD: Lipopolysaccharide-induced microglial activation and neuroprotection against experimental brain injury is independent of hematogenous TLR4. J Neurosci 2012, 34:11706-11715

24. Pascual O, Ben Achour S, Rostaing P, Triller A, Bessis A: Microglia activation triggers astrocyte-mediated modulation of excitatory neurotransmission. Proc Natl Acad Sci U S A 2012, 4:197-205

25. Tremblay S, Miloudi K, Chaychi S, Favret S, Binet F, Polosa A, Lachapelle P, Chemtob S, Sapieha P: Systemic inflammation perturbs developmental retinal angiogenesis and neuroretinal function. Invest Ophthalmol Vis Sci 2013, 13:8125-8139

26. Fan R, Xu F, Previti ML, Davis J, Grande AM, Robinson JK, Van Nostrand WE: Minocycline reduces microglial activation and improves behavioral deficits in a transgenic model of cerebral microvascular amyloid. J Neurosci 2007, 12:3057-3063

27. Krady JK, Basu A, Allen CM, Xu Y, LaNoue KF, Gardner TW, Levison SW: Minocycline reduces proinflammatory cytokine expression, microglial activation, and caspase- 3 activation in a rodent model of diabetic retinopathy. Diabetes 2005, 5:1559-1565

28. Yrjänheikki J, Tikka $T$, Keinänen $R$, Goldsteins $G$, Chan $P H$, Koistinaho J: A tetracycline derivative, minocycline, reduces inflammation and protects against focal cerebral ischemia with a wide therapeutic window. Proc Natl Acad Sci U S A 1999, 23: 13496-13500

29. Milner R, Campbell IL: The extracellular matrix and cytokines regulate microglial integrin expression and activation. J Immunol 2003, 170: $3850-3858$

30. Mosher KI, Andres RH, Fukuhara T, Bieri G, Hasegawa-Moriyama M, He Y, Guzman R, Wyss-Coray T: Neural progenitor cells regulate microglia functions and activity. Nat Neurosci 2012, 15:1485-1487

31. Graber DJ, Hickey WF, Harris BT: Progressive changes in microglia and macrophages in spinal cord and peripheral nerve in the transgenic rat model of amyotrophic lateral sclerosis. J Neuroinflammation 2010, $7: 1-12$

32. Spampanato C, De Leonibus E, Dama P, Gargiulo A, Fraldi A, Sorrentino NC, Russo F, Nusco E, Auricchio A, Surace EM, Ballabio A: Efficacy of a combined intracerebral and systemic gene delivery approach for the treatment of a severe lysosomal storage disorder. Mol Ther 2011, 5:860-869

33. Lee S, Varvel NH, Konerth ME, Xu G, Cardona AE, Ransohoff RM, Lamb BT: CX3CR1 deficiency alters microglial activation and reduces beta-amyloid deposition in two Alzheimer's disease mouse models. Am J Pathol 2010, 5:2549-2562

34. Shaw LM, Messier JM, Mercurio AM: The activation dependent adhesion of macrophages to laminin involves cytoskeletal anchoring and phosphorylation of the alpha6betal integrin. J Cell Biol 1990, 110: $2167-2174$

35. Arnold TD, Ferrero GM, Qiu H, Phan IT, Akhurst RJ, Huang EJ, Reichardt LF: Defective retinal vascular endothelial cell development as a consequence of impaired integrin $\alpha \mathrm{V} \beta 8$-mediated activation of transforming growth factor- $\beta$. J Neurosci 2012, 32:1197-1206

36. Bain JM, Ziegler A, Yang Z, Levison SW, Sen E: TGFBetal stimulates the over-production of white matter astrocytes from precursors of the "brain marrow" in a rodent model of neonatal encephalopathy. PLoS One 2010, 5:e9567

37. Zeng HY, Green WR, Tso MO: Microglial activation in human diabetic retinopathy. Arch Ophthalmol 2008, 126:227-232

38. Rivera JC, Sitaras N, Noueihed B, Hamel D, Madaan A, Zhou T, Honoré JC, Quiniou C, Joyal JS, Hardy P, Sennlaub F, Lubell W, Chemtob S: Microglia and interleukin- $1 \beta$ in ischemic retinopathy elicit microvascular degeneration through neuronal semaphorin-3A. Arterioscler Thromb Vasc Biol 2013, 33:1881-1891 